\title{
Radiative corrections to light neutrino masses in low scale type I seesaw scenarios and neutrinoless double beta decay
}

\author{
J. Lopez-Pavon, ${ }^{a}$ E. Molinaro ${ }^{b}$ and S.T. Petcov ${ }^{a, c, 1}$ \\ ${ }^{a}$ SISSA and INFN - sezione di Trieste, \\ via Bonomea 265, 34136 Trieste, Italy \\ ${ }^{b} C P^{3}$-Origins and Danish Institute for Advanced Study, University of Southern Denmark, \\ Campusvej 55, DK-5230 Odense M, Denmark \\ ${ }^{c}$ Kavli IPMU (WPI), University of Tokyo, \\ 5-1-5 Kashiwanoha, 277-8583 Kashiwa, Japan \\ E-mail: jlpavon@sissa.it, molinaro@cp3.dias.sdu.dk, petcov@sissa.it
}

ABSTRACT: We perform a detailed analysis of the one-loop corrections to the light neutrino mass matrix within low scale type I seesaw extensions of the Standard Model and their implications in experimental searches for neutrinoless double beta decay. We show that a sizable contribution to the effective Majorana neutrino mass from the exchange of heavy Majorana neutrinos is always possible, provided one requires a fine-tuned cancellation between the tree-level and one-loop contribution to the light neutrino masses. We quantify the level of fine-tuning as a function of the seesaw parameters and introduce a generalisation of the Casas-Ibarra parametrization of the neutrino Yukawa matrix, which easily allows to include the one-loop corrections to the light neutrino masses.

Keywords: Neutrino Physics, Rare Decays, Beyond Standard Model

ARXIV EPRINT: 1506.05296

\footnotetext{
${ }^{1}$ Also at: Institute of Nuclear Research and Nuclear Energy, Bulgarian Academy of Sciences, 1784 Sofia, Bulgaria.
} 


\section{Contents}

1 Introduction 1

$2 \quad$ Large active-sterile neutrino mixing and $0 \nu \beta \beta$ decay 2

2.1 Casas-Ibarra parametrization and large active-sterile neutrino mixing 4

$\begin{array}{lll}2.2 & \text { Comparison with extended and inverse seesaw scenarios } & 8\end{array}$

3 One-loop corrections to the neutrino mass matrix 11

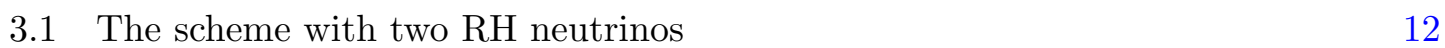

3.2 One-loop generalisation of the Casas-Ibarra parametrization $\quad 14$

$\begin{array}{lll}3.3 & \text { Radiative corrections to the ESS and ISS scenarios } & 17\end{array}$

4 Large heavy neutrino contribution to $0 \nu \beta \beta$ decay 17

$\begin{array}{llr}5 & \text { Conclusions } & 22\end{array}$

A $m_{\nu}^{1-\text { loop in an arbitrary basis for } 2 \text { RH neutrinos }} 23$

\section{Introduction}

The main goal of this work is to study in detail under which conditions the right-handed $(\mathrm{RH})$ neutrinos present in a general type I seesaw scenario [1-4] can give a direct sizable contribution to the neutrinoless double beta $(0 \nu \beta \beta)$ decay rate, i.e., a contribution in the range of sensitivity of the current and upcoming $0 \nu \beta \beta$ decay experiments, once all the relevant constraints are included in the analysis.

In $[5,6]$, it was shown that a sizable sterile neutrino contribution to the $0 \nu \beta \beta$ decay can be achieved if the heavy neutrino spectrum is hierarchical, with at least one RH neutrino with mass $M$ below $100 \mathrm{MeV}$ and the other state(s) above this scale. However, this spectrum is disfavoured by cosmological observations since the region $M \in[1 \mathrm{eV}, 100 \mathrm{MeV}]$ is excluded by BBN and CMB data [7, 8]. In [9-11] the possibility of having a relevant contribution from heavy $\mathrm{RH}$ neutrinos up to the $\mathrm{TeV}$ scale was explored. ${ }^{1}$ It was found that indeed $\mathrm{RH}$ neutrinos as heavy as $100 \mathrm{GeV}-10 \mathrm{TeV}$ could, in principle, give a sizable and observable contribution to the $0 \nu \beta \beta$ decay rate. In [11] the role of the fine-tuning and one-loop effects were discussed, concluding that for $\mathrm{RH}$ neutrino masses above $10 \mathrm{GeV}$ a relatively high level of fine-tuning would be required. In [6] a more detailed study of the one-loop effects was performed and it was found that indeed they are significant and can

\footnotetext{
${ }^{1}$ The interplay between the light and heavy Majorana neutrino contributions in $0 \nu \beta \beta$ decay was investigated phenomenologically first in [12].
} 
play a very important role in the type I seesaw scenario. The lepton number violation introduced through the $\mathrm{RH}$ neutrino Majorana mass term, required to obtain a sizable effect in the $0 \nu \beta \beta$ decay rate, naturally appears at one-loop level in the light neutrino sector. If fine-tuning is not invoked, the light neutrino mass constraints on the one-loop corrections make it very difficult to obtain a significant $(\mathrm{RH})$ heavy Majorana neutrino contribution in the $0 \nu \beta \beta$ decay effective Majorana mass, i.e., to have $\left|m_{\beta \beta}^{\text {heavy }}\right| \gtrsim 0.01 \mathrm{eV}, m_{\beta \beta}^{\text {heavy }}$ being the heavy Majorana neutrino contribution under discussion. We will show, in particular, that the scenario in which $\mathrm{RH}$ neutrinos with a mass $M \gtrsim 1 \mathrm{GeV}$ can give a sizable contribution to the $0 \nu \beta \beta$ decay rate necessarily involves a fine-tuned cancellation between the tree-level and one-loop light neutrino contributions.

More specifically, in this work we re-analyse the conditions under which the heavy Majorana neutrinos with masses $M>100 \mathrm{MeV}$ of the type I seesaw scenario can give a significant direct contribution to the $0 \nu \beta \beta$ decay effective Majorana mass, i.e., a contribution in the range of sensitivity of the current and upcoming $0 \nu \beta \beta$ decay experiments. We show that for $M \gtrsim$ a few $\mathrm{GeV}$ this requires a relatively large active-sterile neutrino mixing (charged current couplings of the heavy Majorana neutrinos). We clarify which seesaw realisations can provide the requisite mixing. We discuss the impact of the one-loop corrections in the different type I seesaw realisations considered. We analyse also numerically the problem of the sizable heavy Majorana neutrino contribution to the $0 \nu \beta \beta$ decay effective Majorana mass, by studying the full parameter space, including the relevant oneloop corrections and the bounds on the active-sterile neutrino mixing from direct searches, charged lepton flavour violation and non-unitarity [13-20]. We quantify, in particular, the level of fine-tuning required in order to have a sizable heavy neutrino contribution to the $0 \nu \beta \beta$ decay rate. In order to do the analysis and generate the right pattern for the light neutrino masses and mixing, we have constructed a modification of the Casas-Ibarra parametrization [21], which takes into account the impact of the one-loop corrections.

The paper is organized as follows: in section 2 we derive under which conditions it is possible to obtain a sizable active-sterile neutrino mixing, which can strongly affect the effective Majorana neutrino mass, $m_{\beta \beta}$. In section 3 we study the impact on $m_{\beta \beta}$ of the one-loop corrections to the light neutrino masses and present our modified CasasIbarra parametrization which takes into account the one-loop effects. In section 4 we perform the numerical analysis and quantify the level of fine-tuning necessary to have a dominant contribution in $m_{\beta \beta}$ from the exchange of the heavy (sterile) neutrinos. Finally, we summarise our results in the concluding section.

\section{Large active-sterile neutrino mixing and $0 \nu \beta \beta$ decay}

We consider the most general type I seesaw scenario [1-4] with $n \geq 2 \mathrm{RH}$ neutrino fields $\nu_{s R}(s=1, \ldots, n)$. After the spontaneous breaking of the electroweak (EW) symmetry the full neutrino mass Lagrangian is

$$
\mathcal{L}_{\nu}=-\overline{\nu_{\ell L}}\left(m_{D}\right)_{\ell s} \nu_{s R}-\frac{1}{2} \overline{\nu_{s L}^{c}}\left(M_{R}\right)_{s t} \nu_{t R}+\text { h.c. }
$$


where $\ell=e, \mu, \tau$ and $\nu_{s L}^{c} \equiv C{\overline{\nu_{s R}}}^{T}, C$ being the charge conjugation matrix. $M_{R}=\left(M_{R}\right)^{T}$ is the Majorana mass matrix of the $\mathrm{RH}$ neutrinos and $m_{D}$ is the $3 \times n$ neutrino Dirac mass matrix. The full mass matrix derived from Lagrangian (2.1) is therefore

$$
\mathcal{M} \equiv\left(\begin{array}{cc}
\mathbf{O} & m_{D} \\
m_{D}^{T} & M_{R}
\end{array}\right)=U^{*} \operatorname{diag}\left(m_{i}, M_{k}\right) U^{\dagger}
$$

where $m_{i}(i=1,2,3)$ and $M_{k}(k=1, \ldots, n)$ are the light and heavy Majorana neutrino masses, respectively. We define $\mathbf{O}$ as a $3 \times 3$ matrix with all elements equal to zero. The full neutrino mass $\mathcal{M}$ is diagonalised by a $(3+n) \times(3+n)$ unitary matrix $U$, through a well known rotation between the neutrino flavour and mass eigenstates. We give below the relation between the left-handed (LH) components of the corresponding fields $\left(\nu_{\ell L}, \nu_{s L}^{c}\right.$ and $\left.\chi_{i L}, N_{k L}\right)$ :

$$
\left(\begin{array}{c}
\nu_{\ell L} \\
\nu_{s L}^{c}
\end{array}\right)=U\left(\begin{array}{c}
\chi_{i L} \\
N_{k L}
\end{array}\right)
$$

Taking into account that the active block of $U$ is unitary to a very good approximation, the complete mixing matrix can be expanded $\mathrm{as}^{2}$

$$
U=\left(\begin{array}{cc}
1-\theta \theta^{\dagger} / 2 & \theta \\
-\theta^{\dagger} & 1-\theta^{\dagger} \theta / 2
\end{array}\right)\left(\begin{array}{cc}
U_{\mathrm{PMNS}} & 0 \\
0 & V
\end{array}\right)+\mathcal{O}\left(\theta^{3}\right)=\left(\begin{array}{cc}
U_{\mathrm{PMNS}} & \theta V \\
-\theta^{\dagger} U_{\mathrm{PMNS}} & V
\end{array}\right)+\mathcal{O}\left(\theta^{2}\right)
$$

where $\theta$ is a $3 \times n$ matrix with "small" entries, which characterises the mixing between the active and the sterile neutrinos, $U_{\mathrm{PMNS}}$ is the PMNS neutrino mixing matrix $[22,23]$ and $V$ is a $n \times n$ unitary matrix. The quantity $(\theta V)_{\ell k}, \ell=e, \mu, \tau, k=1, \ldots, n$, is the coupling of the heavy Majorana neutrino $N_{k}$ to the charged lepton $\ell$ in the weak charged lepton current, and to the flavour neutrino $\nu_{\ell}$ in the weak neutral lepton current.

From the diagonalization of the complete neutrino mass matrix $\mathcal{M}$, at leading order in $\theta$ we have [9]

$$
\begin{aligned}
\theta^{*} M_{R} \theta^{\dagger} & \approx-U_{\mathrm{PMNS}}^{*} \hat{m} U_{\mathrm{PMNS}}^{\dagger} \\
\theta^{*} M_{R} & \approx m_{D} \\
M_{R} & \approx V^{*} \hat{M} V^{\dagger}
\end{aligned}
$$

where

$$
\hat{m} \equiv \operatorname{diag}\left(m_{1}, m_{2}, m_{3}\right), \quad \hat{M} \equiv \operatorname{diag}\left(M_{1}, \ldots, M_{n}\right) .
$$

It follows from eqs. (2.5) and (2.7) that

$$
(\theta V)^{*} \hat{M}(\theta V)^{\dagger} \approx-U_{\mathrm{PMNS}}^{*} \hat{m} U_{\mathrm{PMNS}}^{\dagger} \cdot
$$

In terms of the seesaw parameters we have for the active-sterile neutrino mixing:

$$
\theta^{*} \approx m_{D} M_{R}^{-1}
$$

\footnotetext{
${ }^{2}$ In the following we work in the basis in which the charged lepton mass matrix is diagonal.
} 
Using eqs. (2.5) and (2.10), we recover the usual type I seesaw relation for the (tree-level) light neutrino mass matrix, namely

$$
m_{\nu}^{\text {tree }}=-m_{D} M_{R}^{-1} m_{D}^{T} \equiv-\theta^{*} M_{R} \theta^{\dagger}=-(\theta V)^{*} \hat{M}(\theta V)^{\dagger}=U_{\mathrm{PMNS}}^{*} \hat{m} U_{\mathrm{PMNS}}^{\dagger} .
$$

The effective Majorana neutrino mass, $m_{\beta \beta}$, which enters in the $0 \nu \beta \beta$ decay amplitude, receives, in general, two different contributions, corresponding to the exchanges of the light and heavy virtual Majorana neutrinos:

$$
m_{\beta \beta}=m_{\beta \beta}^{\text {light }}+m_{\beta \beta}^{\text {heavy }},
$$

with

$$
m_{\beta \beta}^{\text {light }}=\sum_{i=1}^{3}\left(U_{\mathrm{PMNS}}\right)_{e i}^{2} m_{i}=-\sum_{k}(\theta V)_{e k}^{2} M_{k},
$$

where we have used eq. (2.9), which holds at tree-level in the type I seesaw models. A good estimate for the contribution due to the heavy Majorana neutrino exchange for $M_{k} \gg$ $100 \mathrm{MeV}$ is [5]

$$
m_{\beta \beta}^{\text {heavy }} \approx-\sum_{k}(\theta V)_{e k}^{2} f(A)\left(M_{a} / M_{k}\right)^{2} M_{k},
$$

where $M_{a} \approx 0.9 \mathrm{GeV}$ and $f(A)$ depends on the decaying isotope considered. For, e.g., ${ }^{48} \mathrm{Ca},{ }^{76} \mathrm{Ge},{ }^{82} \mathrm{Se},{ }^{130} \mathrm{Te}$ and ${ }^{136} \mathrm{Xe}$, the function $f(A)$ takes the values $f(A) \approx 0.033,0.079$, $0.073,0.085$ and 0.068 , respectively.

Using eq. (2.14), it is easy to estimate the minimum mixing $(\theta V)_{\min }$ required in order to have a contribution at the aimed sensitivity of the next generation of $0 \nu \beta \beta$ decay experiments, that is $\left|m_{\beta \beta}^{\text {heavy }}\right| \gtrsim 10^{-2} \mathrm{eV}$. In figure 1 we compare this estimate for $(\theta V)_{\text {min }}$ for the ${ }^{76} \mathrm{Ge}$ isotope, $(\theta V)_{\min }^{2} \simeq 1.6 \times 10^{-10} M \mathrm{GeV}^{-1}$ (dashed line), with the naive seesaw scaling suggested by eq. (2.11), $(\theta V)_{\text {naive }}^{2}=\sqrt{\Delta m_{\text {atm }}^{2}} / M \simeq 5 \times 10^{-11} \mathrm{GeV} / M$ (solid line) as a function of the $\mathrm{RH}$ neutrino mass scale $M$ (expressed in units of $\mathrm{GeV}$ ).

From figure 1 it is clear that for RH neutrino masses larger than $\sim 1 \mathrm{GeV}$ a considerable enhancement with respect to the naive seesaw scaling of $\theta V$ is required in order to have a sizable RH neutrino contribution. Obviously, this enhancement increases with the mass of the RH neutrinos. We notice that in the region $M \approx 500 \mathrm{MeV}-1 \mathrm{GeV}$, the naively estimated mixing, $(\theta V)_{\text {naive }}^{2}$, is in the right ballpark. Similar conclusions are valid for $(\theta V)_{\min }^{2}$ and $(\theta V)_{\text {naive }}^{2}$ in the cases of $0 \nu \beta \beta$ decay of other isotopes $\left({ }^{48} \mathrm{Ca},{ }^{82} \mathrm{Se},{ }^{130} \mathrm{Te},{ }^{136} \mathrm{Xe}\right.$, etc. $)$.

\subsection{Casas-Ibarra parametrization and large active-sterile neutrino mixing}

In order to understand under which conditions an enhancement with respect to the naive scaling of the active-sterile mixing (or equivalently, of the charged current couplings of the heavy Majorana neutrinos $\left.(\theta V)_{\ell k}\right)$ can be expected, we employ the Casas-Ibarra parametrization of $\theta V$ [21]. In this parametrization the light neutrino masses and the angles and phases of the PMNS matrix are input parameters, in such a way that the correct light neutrino mixing pattern is always recovered. The Casas-Ibarra parametrization is obtained rewriting eq. (2.5) as

$$
\left( \pm i \hat{m}^{-1 / 2} U_{\mathrm{PMNS}}^{\dagger} \theta V \hat{M}^{1 / 2}\right)\left( \pm i \hat{m}^{-1 / 2} U_{\mathrm{PMNS}}^{\dagger} \theta V \hat{M}^{1 / 2}\right)^{T} \equiv R R^{T}=1
$$




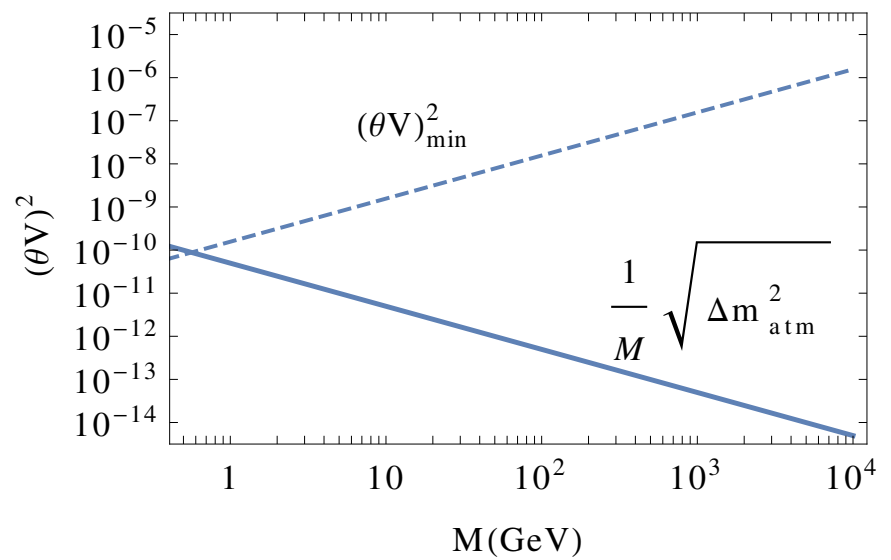

Figure 1. Active-sterile neutrino mixing. The dashed line stands for an estimate of the minimum $(\theta V)^{2}$ required in order to have $\left|m_{\beta \beta}^{\text {heavy }}\right|>10^{-2} \mathrm{eV}$ in the case of $0 \nu \beta \beta$ decay of ${ }^{76} \mathrm{Ge}$. The solid line corresponds to the naive seesaw scaling of $(\theta V)^{2}$ (see the text for further details).

where $R$ is a general $3 \times n$ complex matrix which parametrizes the new physics degrees of freedom associated to the sterile neutrino sector. Using this parametrization, $\theta V$ can be written as

$$
\theta V=\mp i U_{\mathrm{PMNS}} \hat{m}^{1 / 2} R \hat{M}^{-1 / 2}
$$

The matrix $V$ can be set to the unit matrix if one works in the basis in which the Majorana sub-matrix $M_{R}$ is diagonal. ${ }^{3}$

Naively, from eq. (2.11) one may conclude that $\theta V \approx \mathcal{O}\left(\sqrt{\frac{\hat{m}}{\hat{M}}}\right)$, i.e., that the mixing (or coupling) $\theta V$ is expected to be suppressed by the heavy neutrino mass scale. However, having a larger mixing is perfectly possible due to an enhancement factor contained in the matrix $R[9,10]$. Obviously, such enhancement can only be in agreement with the light neutrino spectrum if there is a non-trivial suppression/cancellation in the l.h.s. of eq. (2.9). This extra suppression is related to particular textures of the neutrino mass matrix, which can be motivated, for instance, introducing an extra U(1) global symmetry in the Lagrangian, as it is the case in the so called "inverse" and "direct" seesaw models [26-28]. In these models the indicated global symmetry can be identified with that corresponding to the conservation of a non-standard lepton charge (see further).

In the following we will focus on the minimal seesaw scenario with $n=2 \mathrm{RH}$ sterile neutrinos $^{4}$ (see, e.g., [29-36]) giving rise to two heavy Majorana mass-eigenstate neutrinos, which predicts one massless and two massive light active neutrinos. For the light neutrino

\footnotetext{
${ }^{3}$ An extension of this parametrization to all orders in the seesaw expansion can be found in [24, 25].

${ }^{4}$ In the present article we will use the term "heavy Majorana neutrinos" for Majorana neutrinos having masses exceeding approximately $100 \mathrm{MeV}$.
} 
mass spectrum with normal hierarchy $(\mathrm{NH})$ and inverted hierarchy $(\mathrm{IH})$ we have

$$
\begin{array}{ll}
m_{1}=0, \quad m_{2}=\sqrt{\Delta m_{21}^{2}}, & m_{3}=\sqrt{\Delta m_{31}^{2}}, \\
m_{1}=\sqrt{\left|\Delta m_{32}^{2}\right|-\Delta m_{21}^{2}}, & m_{2}=\sqrt{\left|\Delta m_{32}^{2}\right|}, \quad m_{3}=0 \quad(\mathrm{IH}) .
\end{array}
$$

The current best fit values obtained from the global fit analysis in [37] are

$$
\begin{aligned}
& \Delta m_{21}^{2}=7.50 \times 10^{-5} \mathrm{eV}^{2}, \\
& \Delta m_{31}^{2}=2.457 \times 10^{-3} \mathrm{eV}^{2}(\mathrm{NH}) \text { and } \Delta m_{32}^{2}=-2.449 \times 10^{-3} \mathrm{eV}^{2}(\mathrm{IH}) .
\end{aligned}
$$

In this minimal seesaw scenario, the two (tree-level) non-zero light neutrino masses $m_{2}^{\text {tree }}$ and $m_{3}^{\text {tree }}\left(m_{1}^{\text {tree }}\right)$ in the case of $\mathrm{NH}(\mathrm{IH})$ neutrino mass spectrum satisfy the relation:

$$
m_{2}^{\text {tree }} m_{3(1)}^{\text {tree }} \equiv-\operatorname{det}\left[M_{R}^{-1}\right] \operatorname{det}\left[m_{D}^{T} m_{D}\right], \quad \mathrm{NH}(\mathrm{IH}),
$$

which is basis independent.

In the considered case the $R$-matrix, which enters into eq. (2.16), can be parametrized as [10]

$$
\begin{aligned}
R & =\left(\begin{array}{cc}
0 & 0 \\
\cos \left(\theta_{45}+i \gamma\right) & -\sin \left(\theta_{45}+i \gamma\right) \\
\sin \left(\theta_{45}+i \gamma\right) & \cos \left(\theta_{45}+i \gamma\right)
\end{array}\right), \quad \text { for } \quad \mathrm{NH} \\
R & =\left(\begin{array}{cc}
\cos \left(\theta_{45}+i \gamma\right) & -\sin \left(\theta_{45}+i \gamma\right) \\
\sin \left(\theta_{45}+i \gamma\right) & \cos \left(\theta_{45}+i \gamma\right) \\
0 & 0
\end{array}\right), \quad \text { for } \mathrm{IH},
\end{aligned}
$$

where $\theta_{45}$ and $\gamma$ are real parameters. If $R$ were real, i.e., $\gamma=0$, there is no way to obtain any enhancement of the couplings/mixings $\theta V$ of interest since $R$ would essentially be a real orthogonal matrix. However, for $\gamma \neq 0$ and $e^{ \pm \gamma} \gg 1$ an enhancement of $\theta V$ is possible:

$$
\begin{gathered}
\left|\cos \left(\theta_{45}+i \gamma\right)\right|^{2}=\cos ^{2} \theta_{45}+\sinh ^{2} \gamma \gg 1 \Leftrightarrow e^{ \pm \gamma} \gg 1, \\
\left|\sin \left(\theta_{45}+i \gamma\right)\right|^{2}=\sin ^{2} \theta_{45}+\sinh ^{2} \gamma \gg 1 \Leftrightarrow e^{ \pm \gamma} \gg 1 .
\end{gathered}
$$

In fact, for $e^{ \pm \gamma} \gg 1$ the expression of $R$ in the NH case reduces to

$$
R \approx e^{-i \theta_{45}} \frac{e^{ \pm \gamma}}{2}\left(\begin{array}{cc}
0 & 0 \\
1 & \pm i \\
\mp i & 1
\end{array}\right), \quad \mathrm{NH}
$$

Similarly, one can derive from (2.22) the same limit of $R$ for the IH neutrino mass spectrum:

$$
R \approx e^{-i \theta_{45}} \frac{e^{ \pm \gamma}}{2}\left(\begin{array}{cc}
1 & \pm i \\
\mp i & 1 \\
0 & 0
\end{array}\right), \quad \mathrm{IH} .
$$


Notice that the Casas-Ibarra parameter $\gamma$ in (2.24) and (2.25) can be related to the maximum eigenvalue $y[10]$ of the Dirac mass matrix $m_{D}$ in eq. (2.2), that is

$$
\begin{array}{ll}
y^{2} v^{2}=2 \max \left\{\operatorname{eig}\left(m_{D} m_{D}^{\dagger}\right)\right\}=\frac{1}{2} e^{ \pm \gamma} M_{1}\left(m_{2}+m_{3}\right)(2+z), & \mathrm{NH}, \\
y^{2} v^{2}=2 \max \left\{\operatorname{eig}\left(m_{D} m_{D}^{\dagger}\right)\right\}=\frac{1}{2} e^{ \pm \gamma} M_{1}\left(m_{1}+m_{2}\right)(2+z), & \mathrm{IH},
\end{array}
$$

where $z$ denotes the relative mass splitting of the two heavy Majorana neutrino masses, $z=\left(M_{2}-M_{1}\right) / M_{1}$, and $v=246 \mathrm{GeV}$ is the EW symmetry breaking scale.

Introducing the expression (2.24) (or (2.25)) in eq. (2.16) one obtains [9, 10, 38, 39]

$$
\frac{(\theta V)_{\ell 1}}{(\theta V)_{\ell 2}} \approx \pm i \sqrt{\frac{M_{2}}{M_{1}}} .
$$

Then, in terms of $y$ the active-sterile neutrino mixing in eq. (2.16) takes the form [10]

$$
\begin{aligned}
& \left|(\theta V)_{\ell 1}\right|^{2}=\frac{1}{2(2+z)} \frac{y^{2} v^{2}}{M_{1}^{2}} \frac{m_{3}}{m_{2}+m_{3}}\left|U_{\ell 3}+i \sqrt{m_{2} / m_{3}} U_{\ell 2}\right|^{2}, \quad \mathrm{NH} \\
& \left|(\theta V)_{\ell 1}\right|^{2}=\frac{1}{2(2+z)} \frac{y^{2} v^{2}}{M_{1}^{2}} \frac{m_{2}}{m_{1}+m_{2}}\left|U_{\ell 2}+i \sqrt{m_{1} / m_{2}} U_{\ell 1}\right|^{2}, \quad \mathrm{IH} .
\end{aligned}
$$

All in all, the previous relations imply that in the basis in which the RH neutrino Majorana mass term is diagonal, the neutrino Yukawa couplings, or equivalently $\left(m_{D}\right)_{\ell 1}$ and $\left(m_{D}\right)_{\ell 2}$, should satisfy the following relation:

$$
\frac{\left(m_{D}\right)_{\ell 1}}{\left(m_{D}\right)_{\ell 2}} \approx \pm i \sqrt{\frac{M_{1}}{M_{2}}}
$$

Any texture of the neutrino mass matrix which satisfies this condition gives rise to relatively large couplings $\theta V$ with the right suppression/cancellation in the light (flavour) neutrino mass matrix, which allows to recover the correct light neutrino mass spectrum at tree-level. The relatively large $\theta V$ thus generated can saturate the present bounds even in the case in which the heavy Majorana neutrino spectrum is hierarchical.

Using eqs. (2.14) and (2.28), one can easily estimate the contribution to the $0 \nu \beta \beta$ decay effective Majorana mass due to the exchange of the heavy Majorana neutrinos in the large coupling/mixing case of interest [9]:

$$
m_{\beta \beta}^{\text {heavy }} \approx-(\theta V)_{e 1}^{2} f(A) \frac{M_{a}^{2}}{M_{1}}\left\{1-\left(\frac{M_{1}}{M_{1}+\Delta M}\right)^{2}\right\},
$$

with $^{5} \Delta M=M_{2}-M_{1}$. Clearly, if $\Delta M \ll M_{1}$ the contribution will be proportional to $\Delta M$, while in the limit $\Delta M \gg M_{1}$ the dependence on $\Delta M$ is subleading since the lightest $\mathrm{RH}$ neutrino dominates the contribution.

The interplay between the light and heavy Majorana neutrino exchange contributions in the effective Majorana mass, $m_{\beta \beta}=m_{\beta \beta}^{\text {light }}+m_{\beta \beta}^{\text {heavy }}$, in the scheme under discussion

\footnotetext{
${ }^{5}$ Note that $(\theta V)_{e 1}^{2}$ depends, in particular, on the phase $\theta_{45}$. This implies that $m_{\beta \beta}$ will also depend on $\theta_{45}$ [10].
} 
in which eq. (2.28) holds and $m_{\beta \beta}^{\text {heavy }}$ is given by eq. (2.32), was investigated in detail in [10] in the case when the two heavy Majorana neutrinos form a pseudo-Dirac pair, $0<\Delta M=M_{2}-M_{1} \ll M_{1}, M_{2}$, and have masses in the interval $\sim(50-1000) \mathrm{GeV}$. It was found that there exists a relatively large region of the allowed parameter space of the scheme in which the heavy Majorana neutrino contribution can change drastically the predictions based on the light Majorana neutrino exchange contribution. More specifically, it was found that [10]: i) $\left|m_{\beta \beta}\right|$ in the case of NH spectrum can have values in the interval $0.01 \mathrm{eV} \lesssim\left|m_{\beta \beta}\right| \lesssim 0.1 \mathrm{eV}$, i.e., in the range of sensitivity of the current GERDA [40], EXO [41], Kamland-Zen [42] and CUORE [43] experiments and of a few other experiments under preparation (Majorana [44], SNO+ [45], AMORE [46], etc.). We recall that in the case of $0 \nu \beta \beta$ decay generated only by light Majorana neutrino exchange we have (see, e.g., $[47,48])\left|m_{\beta \beta}\right|=\left|m_{\beta \beta}^{\text {light }}\right| \lesssim 0.005 \mathrm{eV}$;

ii) $\left|m_{\beta \beta}\right|$ in the case of IH spectrum can be strongly suppressed due to partial, or even total, cancellation between $m_{\beta \beta}^{\text {light }}$ and $m_{\beta \beta}^{\text {heavy }}$ in $m_{\beta \beta}$ (see also [49]). Since the magnitude of $m_{\beta \beta}^{\text {heavy }}$, as it follows from eq. (2.32), depends on the atomic number $A$ of the decaying

nucleus [12], the cancellation between $m_{\beta \beta}^{\text {light }}$ and $m_{\beta \beta}^{\text {heavy }}$ in $m_{\beta \beta}$ can take place for a given nucleus (say, e.g., for ${ }^{48} \mathrm{Ca}$ ) but will not hold for other nuclei $\left({ }^{76} \mathrm{Ge},{ }^{82} \mathrm{Se},{ }^{130} \mathrm{Te},{ }^{136} \mathrm{Xe}\right.$, etc.). If the $0 \nu \beta \beta$ decay is due only to the light Majorana neutrino exchange we have in the case of IH spectrum, as is well known [50,51] (see also, e.g., [47]), $0.013 \mathrm{eV} \lesssim\left|m_{\beta \beta}\right|=$ $\left|m_{\beta \beta}^{\text {light }}\right| \lesssim 0.050 \mathrm{eV}$.

On the other hand, in [10] the role of the one-loop corrections was not studied. In [6] it was shown that the one-loop corrections to the light neutrino masses generated in the scheme under discussion turn out to be very relevant. Essentially, a sizable heavy contribution to the $0 \nu \beta \beta$ decay for heavy masses in the range $\sim(50-1000) \mathrm{GeV}$ generates at the same time a very large one-loop correction to the light neutrino masses. In this work we analyse in detail the role of the one-loop effects showing that similar conclusions to the ones drawn in [10] will be obtained. However, we will also show that the price one has to pay in order to have a significant impact of the heavy neutrinos in the $0 \nu \beta \beta$ decay is the requirement of a highly fine-tuned cancellation between the tree-level and one-loop contributions to the light neutrino masses.

\subsection{Comparison with extended and inverse seesaw scenarios}

As an application of the previous results, we consider the effect of heavy $\mathrm{RH}$ neutrinos on the $0 \nu \beta \beta$ decay amplitude in the case of two different realisations of the type I seesaw scenario, which predict a large active-sterile neutrino mixing $\theta V$, that is the well known extended seesaw (ESS) [52] and inverse/direct seesaw (ISS) [26-28] models. In particular, we will clarify how the large mixing realisations described in the previous section in terms of the Casas-Ibarra parametrization match with the ISS and ESS scenarios.

In order to understand the predictions in these classes of models it is useful to adopt the following parametrization of the generic mass terms in the seesaw Lagrangian (2.1), 
namely

$$
\mathcal{M} \equiv\left(\begin{array}{cc}
\mathbf{O} & m_{D} \\
m_{D}^{T} & M_{R}
\end{array}\right)=\left(\begin{array}{ccc}
\mathbf{O} & \mathbf{Y}_{1} v / \sqrt{2} \epsilon \mathbf{Y}_{2} v / \sqrt{2} \\
\mathbf{Y}_{1}^{T} v / \sqrt{2} & \mu^{\prime} & \Lambda \\
\epsilon \mathbf{Y}_{2}^{T} v / \sqrt{2} & \Lambda & \mu
\end{array}\right)
$$

where $\mathbf{Y}_{i} \equiv\left(y_{i e}, y_{i \mu}, y_{i \tau}\right)^{T}$, for $i=1,2$. This parametrization is completely general and, in principle, $\epsilon, \mu, \mu^{\prime}$ and $\Lambda$ can take any value. ${ }^{6}$ However, $\epsilon, \mu$ and $\mu^{\prime}$ can be interpreted as lepton number violating couplings and, therefore, in principle they take arbitrarily small values, because in this case there is an approximate global symmetry of the seesaw Lagrangian corresponding to the conservation of the lepton charge $L^{\prime}=L_{e}+L_{\mu}+L_{\tau}+L_{1}-L_{2}$, where $L_{1}$ and $L_{2}$ are the charges carried by the RH neutrino fields $\nu_{1 R}$ and $\nu_{2 R}$, respectively. In the limit of $\epsilon=\mu=\mu^{\prime}=0$, the conservation of $L^{\prime}$ is exact. In this case the neutrino sector consists of three massless neutrinos and one massive Dirac fermion, which can be inferred, in particular, directly from the expression of the charge $L^{\prime}$ in terms of the charges $L_{\ell}$ and $L_{1,2}[53,54]$. The exact conservation of $L^{\prime}$ corresponds to the case in which condition (2.28) is exactly fulfilled and the RH neutrino splitting satisfies: $\Delta M=M_{2}-M_{1} \rightarrow 0$.

In terms of the new parameters, the exact (tree-level) expression of the light neutrino mass matrix given in (2.11) is proportional to $\mu$ and $\epsilon$, that is

$$
m_{\nu}^{\text {tree }}=\frac{v^{2}}{2\left(\Lambda^{2}-\mu^{\prime} \mu\right)}\left(\mu \mathbf{Y}_{1} \mathbf{Y}_{1}^{T}+\epsilon^{2} \mu^{\prime} \mathbf{Y}_{2} \mathbf{Y}_{2}^{T}-\Lambda \epsilon\left(\mathbf{Y}_{2} \mathbf{Y}_{1}^{T}+\mathbf{Y}_{1} \mathbf{Y}_{2}^{T}\right)\right)
$$

and thus if $\mu=\epsilon=0$ there is a complete cancellation at tree-level for the light neutrino masses. As we will see in the next section, if $\mu^{\prime}$ is different from zero, at least one neutrino mass can be generated at one-loop, even for $\mu=\epsilon=0$ [6]. Furthermore, from the diagonalization of (2.34), we obtain for the product of the smallest $\left(m_{l}^{\text {tree }}\right)$ and the largest $\left(m_{h}^{\text {tree }}\right)$ light neutrino masses:

$$
\begin{aligned}
& \left|m_{l}^{\text {tree }} m_{h}^{\text {tree }}\right|=\left|\operatorname{det}\left[M_{R}^{-1}\right] \operatorname{det}\left[m_{D}^{T} m_{D}\right]\right|= \\
& \frac{v^{4} \epsilon^{2}\left|y_{2 e}^{2}\left(y_{1 \mu}^{2}+y_{1 \tau}^{2}\right)+y_{1 e}^{2}\left(y_{2 \mu}^{2}+y_{2 \tau}^{2}\right)-2 y_{1 e} y_{2 e}\left(y_{1 \mu} y_{2 \mu}+y_{1 \tau} y_{2 \tau}\right)+\left(y_{2 \mu} y_{1 \tau}-y_{1 \mu} y_{2 \tau}\right)^{2}\right|}{4\left|\Lambda^{2}-\mu \mu^{\prime}\right|} .
\end{aligned}
$$

From this relation it follows that in order to have two massive active neutrinos at tree-level, i.e., $\left.m_{l, h}^{\text {tree }} \neq 0, i\right)$ an explicit breaking of the lepton charge conservation via the neutrino Yukawa couplings is necessary, that is the parameter $\epsilon$ must always be different from zero; ii) the vectors of neutrino Yukawa couplings $\mathbf{Y}_{1}$ and $\mathbf{Y}_{2}$ cannot be proportional.

Accordingly, the two seesaw limits of eq. (2.34) which give rise to large active-sterile neutrino mixing $\theta V$ and generate sufficiently small active neutrino masses are:

- i) $\mu^{\prime} \gg \Lambda, y_{1 \alpha} v \gg \mu, \epsilon y_{2 \alpha} v$ (ESS limit). This limit matches the so-called extended seesaw [52] models and corresponds to a hierarchical spectrum for the heavy neutri-

\footnotetext{
${ }^{6}$ In the following we will assume for simplicity that all the parameters introduced in eq. (2.33) are real.
} 
nos:

$$
\begin{aligned}
& M_{1} \approx\left(\Lambda^{2} / \mu^{\prime}-\mu\right), \quad(\theta V)_{\ell 1} \approx i \frac{v}{\sqrt{2} M_{1}}\left[y_{1 \ell} \frac{\Lambda}{\mu^{\prime}-\mu}-\epsilon y_{2 \ell}\left(1-\frac{\Lambda^{2}}{2\left(\mu^{\prime}-\mu\right)^{2}}\right)\right], \\
& M_{2} \approx \mu^{\prime}+\Lambda^{2} / \mu^{\prime}, \quad(\theta V)_{\ell 2} \approx \frac{v}{\sqrt{2} M_{2}}\left[y_{1 \ell}\left(1-\frac{\Lambda^{2}}{2\left(\mu^{\prime}-\mu\right)^{2}}\right)+\epsilon y_{2 \ell} \frac{\Lambda}{\mu^{\prime}-\mu}\right],
\end{aligned}
$$

where we also show the corresponding mixing with the active neutrinos. Then, the approximate tree-level contribution to the $0 \nu \beta \beta$ decay effective Majorana mass due to the exchange of the light and the heavy neutrinos is

$$
\begin{aligned}
m_{\beta \beta}^{\text {light }} & \approx \frac{v^{2}}{2\left(\Lambda^{2} / \mu^{\prime}-\mu\right)}\left(\frac{\mu}{\mu^{\prime}} y_{1 e}^{2}-2 \epsilon \frac{\Lambda}{\mu^{\prime}} y_{1 e} y_{2 e}\right), \\
m_{\beta \beta}^{\text {heavy }} & \approx f(A) \frac{v^{2} M_{a}^{2}}{2\left(\Lambda^{2} / \mu^{\prime}-\mu\right)^{3}}\left(\frac{\Lambda^{2}}{\mu^{\prime 2}} y_{1 e}^{2}-2 \epsilon \frac{\Lambda}{\mu^{\prime}} y_{1 e} y_{2 e}\right),
\end{aligned}
$$

respectively. The dominant term in $m_{\beta \beta}^{\text {heavy }}$ is due to the exchange of the lighter of the two heavy Majorana neutrinos $N_{1}$, the exchange of $N_{2}$ giving a subleading (and negligible in the leading approximation we employed) correction. Notice that, if $\Lambda^{2} / \mu^{\prime} \gg \mu, m_{\beta \beta}^{\text {light }}$ becomes independent of $\mu^{\prime}$ while $m_{\beta \beta}^{\text {heavy }}$ is proportional to $\mu^{\prime}$ :

$$
\begin{aligned}
m_{\beta \beta}^{\text {light }} & \approx \frac{v^{2}}{2 \Lambda^{2}}\left(\mu y_{1 e}^{2}-2 \epsilon \Lambda y_{1 e} y_{2 e}\right), \\
m_{\beta \beta}^{\text {heavy }} & \approx f(A) \frac{\mu^{\prime} v^{2} M_{a}^{2}}{2 \Lambda^{4}}\left(y_{1 e}^{2}-2 \epsilon \frac{\mu^{\prime}}{\Lambda} y_{1 e} y_{2 e}\right) .
\end{aligned}
$$

- ii) $\Lambda \gg y_{1 \alpha} v \gg \mu^{\prime}, \mu, \epsilon y_{2 \alpha} v$ (ISS limit). This limit corresponds to a minimal realisation with only two RH neutrinos of the so-called inverse or direct seesaw models [39]. In this case the heavy neutrino spectrum is quasi-degenerate, forming a quasi-Dirac pair $[55,56]$

$$
\begin{aligned}
& M_{1} \approx \Lambda-\frac{\mu+\mu^{\prime}}{2}, \quad(\theta V)_{\ell 1} \approx i \frac{v}{2 M_{1}}\left[y_{1 \ell}\left(1+\frac{\mu-\mu^{\prime}}{4 \Lambda}\right)-\epsilon y_{2 \ell}\left(1-\frac{\mu-\mu^{\prime}}{4 \Lambda}\right)\right], \\
& M_{2} \approx \Lambda+\frac{\mu+\mu^{\prime}}{2}, \quad(\theta V)_{\ell 2} \approx \frac{v}{2 M_{2}}\left[y_{1 \ell}\left(1-\frac{\mu-\mu^{\prime}}{4 \Lambda}\right)+\epsilon y_{2 \ell}\left(1+\frac{\mu-\mu^{\prime}}{4 \Lambda}\right)\right],
\end{aligned}
$$

In this limit the light and heavy contributions to the $0 \nu \beta \beta$ decay rate are given by:

$$
\begin{aligned}
m_{\beta \beta}^{\text {light }} & \approx \frac{v^{2}}{2 \Lambda^{2}}\left(\mu y_{1 e}^{2}-2 \epsilon \Lambda y_{1 e} y_{2 e}\right), \\
m_{\beta \beta}^{\text {heavy }} & \approx f(A) \frac{v^{2} M_{a}^{2}}{2 \Lambda^{4}}\left(\left(2 \mu+\mu^{\prime}\right) y_{1 e}^{2}-2 \epsilon \Lambda y_{1 e} y_{2 e}\right) .
\end{aligned}
$$

Both of them are proportional to the small lepton number violating parameters, as it should be. Notice that the expression of $m_{\beta \beta}^{\text {light }}$ above is exactly the same as the one given in eq. (2.40). 
On one hand, it follows from eqs. (2.40), (2.41), (2.44) and (2.45) that a relatively large contribution to the $0 \nu \beta \beta$ decay rate due to the heavy Majorana neutrino exchange might be possible at tree-level without affecting the smallness of the light neutrino masses since in the limits considered here $m_{\beta \beta}^{\text {heavy }} \propto \mu^{\prime}$, while $m_{\beta \beta}^{\text {light }}$ is independent of $\mu^{\prime}$. On the other hand, eqs. (2.36)-(2.37) and (2.42)-(2.43) confirm that the condition to obtain relatively large mixings, eq. (2.28), is fulfilled at leading order, that is in the Casas-Ibarra parametrization the $R$-matrix corresponding to these two cases is similar to the textures reported in eqs. (2.24) and (2.25).

Finally, we note that in the case of the ISS model, the smallness of the light neutrino masses comes from the existence of an approximate symmetry corresponding to the conservation of the lepton charge $L^{\prime}$. In contrast, in the ESS limit, the conservation of the lepton charge $L^{\prime}$ is strongly violated through the $\mu^{\prime}$ coupling. This means that, in principle, the one-loop corrections to the neutrino masses can be expected to be more important in the ESS limit than in the ISS one since in the ESS case there is no symmetry protecting the light neutrino masses from getting relatively large corrections [6].

\section{One-loop corrections to the neutrino mass matrix}

We turn now to the computation of the one-loop corrections to the light neutrino mass matrix and the effective Majorana neutrino mass associated to $0 \nu \beta \beta$ decay amplitude.

At one-loop the neutrino self-energy $\Sigma(p)$ provides the dominant finite correction to $m_{\nu}[6,57-60]$, which depends on the square of the neutrino Yukawa couplings, as in the tree-level contribution (2.11), and is further suppressed by the one-loop factor $1 /\left(16 \pi^{2}\right)$. In a generic basis, with the Dirac and Majorana mass terms defined in Lagrangian (2.1), we obtain:

$$
\mathcal{M}=\left(\begin{array}{cc}
m_{\nu}^{1-\text { loop }} & m_{D} \\
m_{D}^{T} & M_{R}
\end{array}\right)=U^{*} \operatorname{diag}\left(m_{i}, M_{k}\right) U^{\dagger},
$$

where the new Majorana mass term generated at one-loop is in this case

$$
m_{\nu}^{1-\text { loop }}=\frac{1}{(4 \pi v)^{2}} m_{D}\left(M_{R}^{-1} F\left(M_{R} M_{R}^{\dagger}\right)+F\left(M_{R}^{\dagger} M_{R}\right) M_{R}^{-1}\right) m_{D}^{T} .
$$

The loop function $F(x)$ is defined as

$$
F(x) \equiv \frac{x}{2}\left(3 \log \left(x / M_{Z}^{2}\right)\left(x / M_{Z}^{2}-1\right)^{-1}+\log \left(x / M_{H}^{2}\right)\left(x / M_{H}^{2}-1\right)^{-1}\right),
$$

$M_{H}$ and $M_{Z}$ denoting the Higgs and the $Z$ boson mass, respectively. Hence, the overall light neutrino mass matrix, $m_{\nu}$, is given by the sum of the tree-level (2.11) and one-loop (3.2) terms, which in the basis of charged lepton mass eigenstates satisfies the relation

$$
m_{\nu}=m_{\nu}^{\text {tree }}+m_{\nu}^{1-\text { loop }}=U_{\mathrm{PMNS}}^{*} \operatorname{diag}\left(m_{1}, m_{2}, m_{3}\right) U_{\mathrm{PMNS}}^{\dagger} \cdot
$$

The finite radiative correction given in (3.2) is in general subdominant in the case of $\mathrm{RH}$ neutrinos with a high mass scale $M \gg v$, but it may be sizable and comparable to the tree-level term in seesaw scenarios where the lepton number violating scale is taken below 
the $\mathrm{TeV}$ range. It is therefore interesting to analyse in greater detail the dependence of the light neutrino masses on the additional finite one-loop contribution, eq. (3.2).

In the basis in which the $\mathrm{RH}$ neutrino mass is diagonal, the one-loop correction of interest has the following form:

$$
\left(m_{\nu}^{1-\text { loop }}\right)_{\ell \ell^{\prime}}=\frac{1}{(4 \pi v)^{2}}(\theta V)_{\ell k}^{*} M_{k}^{3}\left(\frac{3 \log \left(M_{k}^{2} / M_{Z}^{2}\right)}{M_{k}^{2} / M_{Z}^{2}-1}+\frac{\log \left(M_{k}^{2} / M_{H}^{2}\right)}{M_{k}^{2} / M_{H}^{2}-1}\right)(\theta V)_{k \ell^{\prime}}^{\dagger},
$$

where we have used eqs. (2.6) and (2.7). The contribution of the one-loop correction under discussion to the effective Majorana neutrino mass $m_{\beta \beta}^{\text {light }}$, generated by the light Majorana neutrino exchange, as can be shown, is given by

$$
m_{\beta \beta}^{1-\text { loop }}=\left(m_{\nu}^{1-\text { loop }}\right)_{e e}^{*} .
$$

\subsection{The scheme with two RH neutrinos}

In the phenomenologically interesting scheme with two RH neutrinos, for each non-zero eigenvalue $m_{k}$ of eq. (3.4), we have the exact relation

$$
\begin{aligned}
0 & =\operatorname{det}\left[m_{k} \mathbf{1}_{\mathbf{3} \times \mathbf{3}}+m_{D} M_{R}^{-1}\left(\mathbf{1}_{\mathbf{2} \times \mathbf{2}}-\mathcal{H}\left(M_{R}\right)\right) m_{D}^{T}\right] \\
& =m_{k} \operatorname{det}\left[m_{k} \mathbf{1}_{\mathbf{2} \times \mathbf{2}}+M_{R}^{-1}\left(\mathbf{1}_{\mathbf{2} \times \mathbf{2}}-\mathcal{H}\left(M_{R}\right)\right) m_{D}^{T} m_{D}\right],
\end{aligned}
$$

where the second equality follows form the Sylvester's determinant theorem and we have introduced the function ${ }^{7}$

$$
\mathcal{H}\left(M_{R}\right) \equiv \frac{1}{(4 \pi v)^{2}}\left(F\left(M_{R} M_{R}^{\dagger}\right)+M_{R} F\left(M_{R}^{\dagger} M_{R}\right) M_{R}^{-1}\right) .
$$

Using (3.7) and (2.20), we get the identity

$$
\operatorname{det}\left[\mathbf{1}_{\mathbf{2} \times \mathbf{2}}-\mathcal{H}\left(M_{R}\right)\right]\left|m_{l}^{\text {tree }} m_{h}^{\text {tree }}\right|=m_{l} m_{h},
$$

where $m_{l}\left(m_{h}\right)$ is the smaller (larger) non-zero active neutrino mass, whose experimental value in the cases of $\mathrm{NH}$ and $\mathrm{IH}$ neutrino mass spectrum is given in eqs. (2.17) and (2.18), respectively. $^{8}$ Therefore, the determinant on the left hand side of eq. (3.9) provides a measurement of the deviation of the tree-level mass eigenvalues from the observed neutrino masses. Notice that, this is a positive quantity smaller than one in the scenarios considered here. As a consequence of eq. (3.9), one has that in the case $m_{l}^{\text {tree }}=0$, i.e. if two of the active neutrinos are massless at tree-level, it is not possible to generate at one-loop level two non-zero light (active) neutrino masses in the spectrum. In other words, in such a scenario both the solar and atmospheric neutrino oscillation mass differences cannot be radiatively generated.

As it is not difficult to show, in the minimal scenario with only two heavy Majorana neutrinos, in which condition (2.28) is exactly fulfilled, the one-loop contribution to the

\footnotetext{
${ }^{7}$ The definition given in eq. (3.8) is by construction basis independent.

${ }^{8}$ In the convention we are using $m_{l}^{\text {tree }} m_{h}^{\text {tree }}=m_{2}^{\text {tree }} m_{3}^{\text {tree }}\left(m_{l}^{\text {tree }} m_{h}^{\text {tree }}=m_{1}^{\text {tree }} m_{2}^{\text {tree }}\right)$ and $m_{l} m_{h}=m_{2} m_{3}$ $\left(m_{l} m_{h}=m_{1} m_{2}\right)$ in the $\mathrm{NH}(\mathrm{IH})$ case.
} 
light neutrino mass matrix goes to zero in the limit $\Delta M=M_{2}-M_{1} \rightarrow 0$. Indeed, from eqs. (2.28) and (3.6) we find:

$$
\begin{aligned}
m_{\beta \beta}^{1-\text { loop }} & =\frac{1}{(4 \pi v)^{2}}(\theta V)_{e 1}^{2} M_{1}^{3}\left\{\left[\left(\frac{3 \log \left(M_{1}^{2} / M_{Z}^{2}\right)}{M_{1}^{2} / M_{Z}^{2}-1}+\frac{\log \left(M_{1}^{2} / M_{H}^{2}\right)}{M_{1}^{2} / M_{H}^{2}-1}\right)-\left(M_{1}^{2} \rightarrow M_{2}^{2}\right)\right]\right. \\
& \left.-z(2+z)\left(\frac{3 \log \left(M_{2}^{2} / M_{Z}^{2}\right)}{M_{2}^{2} / M_{Z}^{2}-1}+\frac{\log \left(M_{2}^{2} / M_{H}^{2}\right)}{M_{2}^{2} / M_{H}^{2}-1}\right)\right\}
\end{aligned}
$$

where $z \equiv \Delta M / M_{1}$, i.e., $M_{2}=(1+z) M_{1}$. Note that eq. (3.10) is valid for arbitrary values of $z$ and $M_{1}$. In the case of $M_{1}^{2}, M_{2}^{2} \ll M_{Z}^{2}, M_{H}^{2}$ we get:

$$
m_{\beta \beta}^{1-\text { loop }}=\frac{(\theta V)_{e 1}^{2}}{(4 \pi v)^{2}} M_{1}^{3}\left[8(1+z)^{2} \log (1+z)+z(2+z)\left(3 \log \left(M_{1}^{2} / M_{Z}^{2}\right)+\log \left(M_{1}^{2} / M_{H}^{2}\right)\right)\right] .
$$

If, in addition, $z \ll 1$, this expression further simplifies to:

$$
m_{\beta \beta}^{1-\text { loop }}=\frac{(\theta V)_{e 1}^{2}}{(4 \pi v)^{2}} M_{1}^{3} z(2+z)\left[4(1+z)^{2}+3 \log \left(M_{1}^{2} / M_{Z}^{2}\right)+\log \left(M_{1}^{2} / M_{H}^{2}\right)\right] .
$$

In the opposite limit, namely, $M_{1}^{2}, M_{2}^{2} \gg M_{Z}^{2}, M_{H}^{2}, m_{\beta \beta}^{1-\text { loop }}$ takes also a rather simple form for $z \ll 1$. In this case, to leading order in $z \ll 1$, we obtain:

$$
m_{\beta \beta}^{1-\text { loop }}=-2 z \frac{1}{(4 \pi v)^{2}}(\theta V)_{e 1}^{2} M_{1}\left(3 M_{Z}^{2}+M_{H}^{2}\right) .
$$

Thus, in the scheme considered here, in which condition (2.28) is fulfilled, the magnitude of the one-loop correction to $m_{\beta \beta}^{\text {light }}$ of interest, $m_{\beta \beta}^{1-\text { loop }}$, exhibits a strong dependence on $z$. This dependence is particularly important in the case when the two heavy Majorana neutrinos form a pseudo-Dirac pair, $0<\Delta M \ll M_{1}, M_{2}$, or $z \ll 1$. In this case the ratio of the one-loop correction to the $0 \nu \beta \beta$ decay amplitude and the heavy Majorana neutrino exchange contribution given in eq. (2.32), $\left|m_{\beta \beta}^{1-\text { loop }} / m_{\beta \beta}^{\text {heavy }}\right|$, practically depends only on the mass $M_{1}$. As it is not difficult to show, for $f(A)=0.79(0.033)$, i.e., for ${ }^{76} \mathrm{Ge}\left({ }^{48} \mathrm{Ca}\right)$, we have $\left|m_{\beta \beta}^{1-\text { loop }} / m_{\beta \beta}^{\text {heavy }}\right| \approx 1$ at $M_{1} \approx 15(9.7) \mathrm{GeV}$. For $M_{1}>15(9.7) \mathrm{GeV}\left(M_{1}<15(9.7) \mathrm{GeV}\right)$, $\left|m_{\beta \beta}^{1-\text { loop }}\right|$ is bigger (smaller) than $\left|m_{\beta \beta}^{\text {heavy }}\right|$. This is illustrated in figure 2 , which shows the dependence of $\left|m_{\beta \beta}^{1-\text { loop }}\right|$ and $\left|m_{\beta \beta}^{\text {heavy }}\right|$ on $M_{1}>0.5 \mathrm{GeV}$ for $\Delta M=10^{-2} \mathrm{GeV}$ in the scheme in which condition (2.28) is exactly fulfilled and fixing the active-sterile mixing to the reference value of $\left|(\theta V)_{e 1}^{2}\right|=10^{-3}$. In this plot the Higgs mass has been set to $M_{H}=125 \mathrm{GeV}$. Note, however, that given the values of $M_{Z}=90 \mathrm{GeV}$ and $M_{H}=125 \mathrm{GeV}$, for $M_{1}=15(9.7) \mathrm{GeV}$, the factor $\left(4(1+z)^{2}+3 \log \left(M_{1}^{2} / M_{Z}^{2}\right)+\log \left(M_{1}^{2} / M_{H}^{2}\right)\right)$ in eq. (3.12) for $m_{\beta \beta}^{1-\text { loop }}$ is negative. Thus, at $M_{1}=15(9.7) \mathrm{GeV}$, we have $m_{\beta \beta}^{1-\text { loop }} / m_{\beta \beta}^{\text {heavy }}>0$ (see eq. (2.32)), and therefore a cancellation, or even a partial compensation, between the two terms $m_{\beta \beta}^{1-\text { loop }}$ and $m_{\beta \beta}^{\text {heavy }}$ in the $0 \nu \beta \beta$ decay amplitude is impossible.

As it should be clear from figure 2 and eqs. (3.11)-(3.13), $\left|m_{\beta \beta}^{1-\text { loop }}\right|$ grows rapidly with the increase of $M_{1}$. However, the dependence of $\left|m_{\beta \beta}^{1-\text { loop }}\right|$ on $z$ when $z \ll 1$ makes 


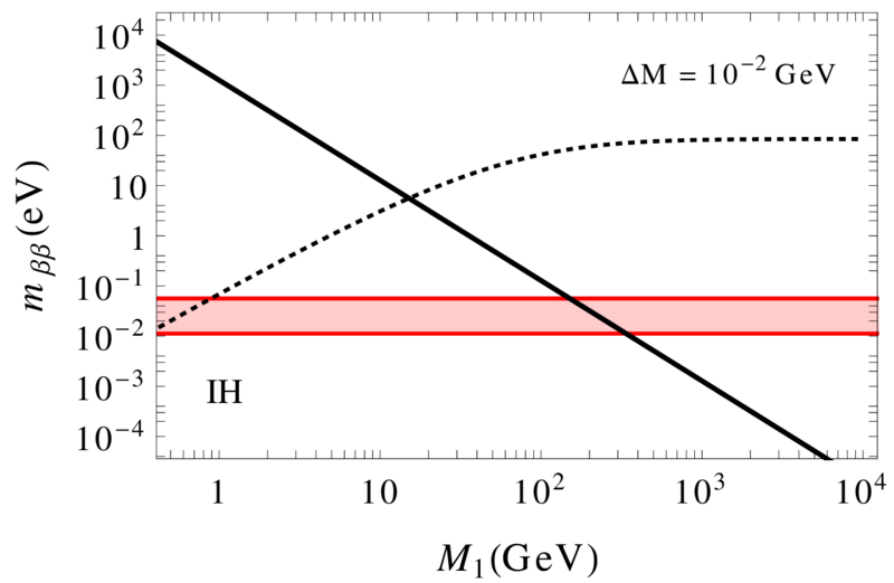

Figure 2. The contributions to the $0 \nu \beta \beta$ decay effective Majorana mass due to the one-loop correction to the light neutrino mass matrix (dashed line) and due to the heavy Majorana neutrino exchange (solid line), $\left|m_{\beta \beta}^{1-\text { loop }}\right|$ and $\left|m_{\beta \beta}^{\text {heavy }}\right|$ (eqs. (3.10) and (2.32)), as functions of the heavy Majorana neutrino mass $M_{1}$, for $\Delta M=10^{-2} \mathrm{GeV},\left|(\theta V)_{e 1}\right|^{2}=10^{-3}$ and $f(A)=0.079$ (i.e., for $\left.{ }^{76} \mathrm{Ge}\right)$. The range of values the effective Majorana neutrino mass can take in the case of light Majorana neutrino exchange and IH spectrum is also shown (the band in red color). See the text for further details.

it possible, in principle, for $\left|m_{\beta \beta}^{1-\text { loop }}\right|$ to have values in the range of sensitivity of the current and next generation of $0 \nu \beta \beta$ decay experiments, i.e., to have $\left|m_{\beta \beta}^{1-\text { loop }}\right| \sim(0.01-$ $0.10) \mathrm{eV}$ even for, e.g., $M_{1}=10^{3} \mathrm{GeV}$ and the maximal value of $\left|(\theta V)_{e 1}^{2}\right|=10^{-3}$ allowed by the current data. This requires, however, exceedingly small values of $z$, which lead to a subleading heavy neutrino contribution. Indeed, using the quoted values of $M_{1}$ and $\left|(\theta V)_{e 1}^{2}\right|$, and taking into account that $v=246 \mathrm{GeV}$, it is not difficult to find from eq. (3.13) that we can have $\left|m_{\beta \beta}^{1-\text { loop }}\right| \approx 0.01(0.10) \mathrm{eV}$ for $z \approx 6 \times 10^{-10}\left(6 \times 10^{-9}\right)$. Such a small value of $z$ suggests a severe fine-tuning, but it can also be understood in the context of the ISS scenario as a technically naturally small value of the lepton number violating parameters of this model.

In the analyses which follow we will not assume that eq. (2.28) relating $(\theta V)_{e 1}$ and $(\theta V)_{e 2}$ is satisfied. We will use only the phenomenological constraint on $(\theta V)_{e 1}$ and $(\theta V)_{e 2}[13-20]$. Notice, however, that for values of the Casas-Ibarra parameter $|\gamma| \gtrsim 6$ (see eqs. (2.16), (2.24) and (2.25)), the relation given in eq. (2.28) is effectively satisfied.

\subsection{One-loop generalisation of the Casas-Ibarra parametrization}

In order to make sure that we generate the correct light neutrino mixing pattern, it is useful to generalise the Casas-Ibarra parametrization introduced in the previous section including the one-loop correction to the neutrino mass matrix. Taking into account the expression (3.5) for $\left(m_{\nu}^{1-\text { loop }}\right)_{\ell \ell^{\prime}}$ in the basis in which the RH neutrino mass is diagonal, 

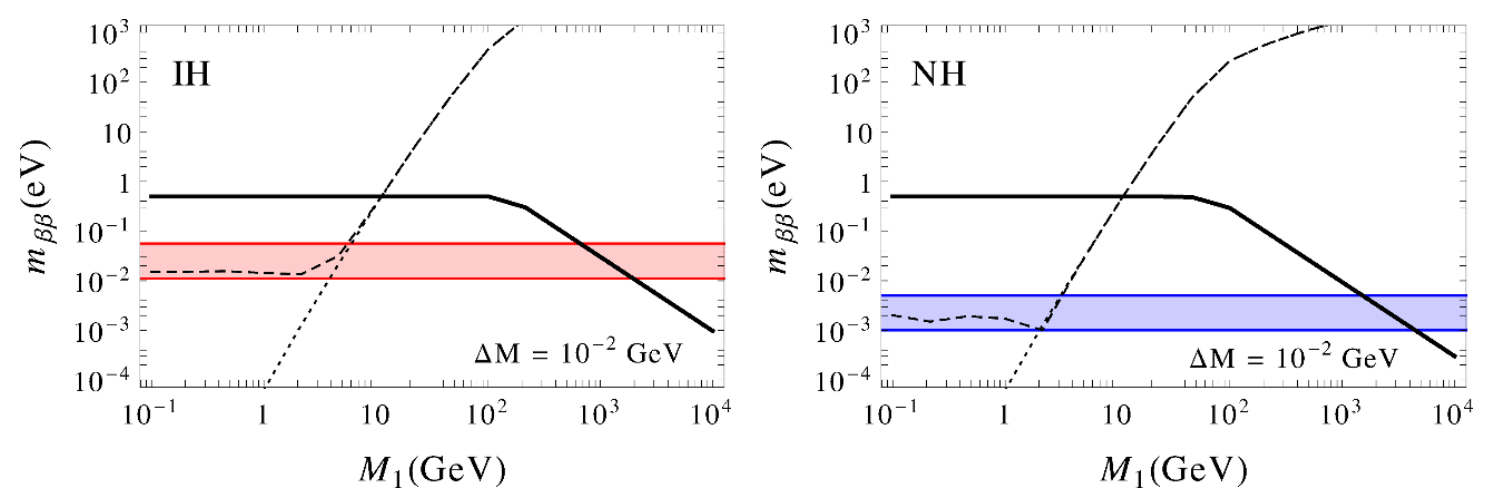

Figure 3. Maximum value of the contribution to the $0 \nu \beta \beta$ decay effective Majorana mass due to the heavy Majorana neutrino exchange $\left|m_{\beta \beta}^{\text {heavy }}\right|$ (solid thick line) for ${ }^{76} \mathrm{Ge}$ and $\Delta M=10^{-2} \mathrm{GeV}$ in the IH (left panel) and $\mathrm{NH}$ (right panel) case, including the following constraints: $\left|m_{\beta \beta}^{\text {heavy }}\right| \leq 0.5 \mathrm{eV}$ and $\left|(\theta V)_{e 1}\right|^{2}+\left|(\theta V)_{e 2}\right|^{2} \leq 2 \times 10^{-3}$. The corresponding values of the contributions to the $0 \nu \beta \beta$ decay effective Majorana mass due to the tree-level (dashed line) and one-loop correction (dotted line) to the light neutrino mass matrix, $\left|m_{\beta \beta}^{\text {tree }}\right|$ and $\left|m_{\beta \beta}^{1-l o o p}\right|$, are also shown. The range of values the effective Majorana mass can take in the case of light Majorana neutrino exchange and $\mathrm{IH}(\mathrm{NH})$ spectrum is shown in the red (blue) band. See the text for further details.

eq. (3.4) takes the explicit form:

$$
\begin{aligned}
\left(m_{\nu}\right)_{\ell \ell^{\prime}} & =-\left(m_{D} V\right)_{\ell k}\left[M_{k}^{-1}-\frac{1}{(4 \pi v)^{2}} M_{k}\left(\frac{3 \log \left(M_{k}^{2} / M_{Z}^{2}\right)}{M_{k}^{2} / M_{Z}^{2}-1}+\frac{\log \left(M_{k}^{2} / M_{H}^{2}\right)}{M_{k}^{2} / M_{H}^{2}-1}\right)\right]\left(V^{T} m_{D}^{T}\right)_{k \ell^{\prime}} \\
& \equiv-\left(m_{D} V\right)_{\ell k} \Delta_{k}^{-1}\left(V^{T} m_{D}^{T}\right)_{k \ell^{\prime}}=\left(U_{\mathrm{PMNS}}^{*} \operatorname{diag}\left(m_{1}, m_{2}, m_{3}\right) U_{\mathrm{PMNS}}^{\dagger}\right)_{\ell \ell^{\prime}} .
\end{aligned}
$$

Hence, in analogy to the tree-level contribution, we have now

$$
\left( \pm i \hat{m}^{-1 / 2} U_{\mathrm{PMNS}}^{\dagger} \theta V \hat{M} \Delta^{-1 / 2}\right)\left( \pm i \hat{m}^{-1 / 2} U_{\mathrm{PMNS}}^{\dagger} \theta V \hat{M} \Delta^{-1 / 2}\right)^{T} \equiv R R^{T}=1 .
$$

Thus, we get the following expression for the heavy Majorana neutrino couplings in the weak charged current, or equivalently, for the active-sterile neutrino mixing, at one-loop order:

$$
\theta V=\mp i U_{\mathrm{PMNS}} \hat{m}^{1 / 2} R \Delta^{1 / 2} \hat{M}^{-1} .
$$

In the numerical analysis reported in section 4 we will make use of this parametrization of $\theta V$, with $R$ given in (2.21) and (2.22), in order to include the one-loop corrections to the light neutrino masses and at the same time ensure that all the neutrino mixing parameters match with their experimental values.

In figure 3 we illustrate the interplay between the contributions to the $0 \nu \beta \beta$ decay effective Majorana neutrino mass due to the heavy Majorana neutrino exchange, $\left|m_{\beta \beta}^{\text {heavy }}\right|$, the tree-level light neutrino masses, $\left|m_{\beta \beta}^{\text {tree }}\right|=\left|\left(m_{\nu}^{\text {tree }}\right)_{e e}^{*}\right|$, and the one-loop correction to the light neutrino mass matrix, $\left|m_{\beta \beta}^{1-\text { loop }}\right|=\left|\left(m_{\nu}^{1-\text { loop }}\right)_{e e}^{*}\right|$, using the generalised Casas-Ibarra parametrization derived above. In particular, we have maximised $\left|m_{\beta \beta}^{\text {heavy }}\right|$ over the free parameters of the model $\left(\theta_{45}, \gamma\right.$ and the Dirac and Majorana phases of the PMNS matrix), 
in order to show the maximum heavy neutrino contribution to the process (solid thick line) as a function of $M_{1}$ for $\Delta M=10^{-2} \mathrm{GeV}$ and fixing the already measured PMNS parameters and neutrino squared mass differences to the best fit values given in [37]. The Higgs mass has been set to $M_{H}=125 \mathrm{GeV}$. In the plot we show the corresponding value of the separate contributions associated to the tree-level (dashed line) and one-loop correction (dotted line) to the light neutrino mass matrix. We also impose the following constraints: $\left|m_{\beta \beta}^{\text {heavy }}\right| \leq 0.5 \mathrm{eV}$ and $\left|(\theta V)_{e 1}\right|^{2}+\left|(\theta V)_{e 2}\right|^{2} \leq 2 \times 10^{-3}$.

From figure 3 we conclude that for $M_{1} \lesssim 1 \mathrm{GeV}$ the one-loop correction is subleading for $\Delta M=10^{-2} \mathrm{GeV}$, being the tree-level contribution the one responsible for the light neutrino mass generation. At the same time, in that region the heavy neutrino contribution to the $0 \nu \beta \beta$ decay effective Majorana neutrino mass can be sizable and larger than the one from light neutrino exchange. According to the estimate given in figure 1 , for $M_{1} \lesssim 1 \mathrm{GeV}$ there is no need of any enhancement of the active-sterile mixing with respect to the naive seesaw scaling in order to obtain a sizable $\left|m_{\beta \beta}^{\text {heavy }}\right|$. However, around $M_{1} \sim 2 \mathrm{GeV}$, the oneloop correction starts to be of the same size as the value of the light neutrino contribution dictated by neutrino oscillation data. Indeed, this correction increases with $M_{1}$ in such a way that in order to stabilise the light neutrino mass and mixing, a fine-tuned cancellation between the tree-level and one-loop correction is required. This is reflected in the fact that for $M_{1} \gtrsim 5 \mathrm{GeV}$ the dotted and dashed lines merge. Therefore, as it is shown in figure 3, for $5 \mathrm{GeV} \lesssim M_{1} \lesssim 1 \mathrm{TeV}$ a sizable $\left|m_{\beta \beta}^{\text {heavy }}\right|$ can in principle be realised, but a fine-tuned cancellation between the tree-level and one-loop contributions to the light neutrino masses is also necessary.

Note that the bound $\left|m_{\beta \beta}^{\text {heavy }}\right| \leq 0.5 \mathrm{eV}$ imposed by us can be saturated for $M_{1} \lesssim$ $100 \mathrm{GeV}$. At $M_{1}=10 \mathrm{GeV}$, for instance, we have $\left|m_{\beta \beta}^{\text {heavy }}\right|=0.5 \mathrm{eV}$ for $\left|(\theta V)_{e 1}\right|^{2}+$ $\left|(\theta V)_{e 2}\right|^{2} \simeq 0.8 \times 10^{-4}$, where we have used $f(A)=0.079$ corresponding to ${ }^{76} \mathrm{Ge}$. For $M_{1} \gtrsim 100 \mathrm{GeV}$ the maximum value of $\left|m_{\beta \beta}^{\text {heavy }}\right|$ decreases with $M_{1}$ since an active-sterile mixing $\left|(\theta V)_{e i}\right|^{2}$ bigger than $2 \times 10^{-3}$ would be required in order to saturate the bound.

It is interesting that the solid line and the blue and red bands in figure 3 intersect around $M_{1} \sim 10^{3} \mathrm{GeV}$. This implies that in the case of NH neutrino mass spectrum, the effective Majorana neutrino mass $\left|m_{\beta \beta}\right|$ can be larger at $0.1 \mathrm{GeV} \lesssim M_{1} \lesssim 10^{3} \mathrm{GeV}$ than that predicted in the case of the light neutrino exchange mechanism. In particular, it can be in the range of sensitivity of the experiments aiming to probe the range of values of the effective Majorana mass corresponding to the IH and quasi-degenerate (QD) light neutrino mass spectra (see, e.g., [47]). In the case of the IH light neutrino mass spectrum, the indicated result implies that at $M_{1} \lesssim 10^{3} \mathrm{GeV}$ there can be, in principle, a significant interplay between the light and heavy Majorana neutrino exchange contributions in the effective Majorana mass, as discussed in detail in [10] and summarised by us at the end of subsection II.A (see the paragraph before the last in subsection II.A). More specifically, due to this interplay of the light and heavy Majorana neutrino contributions, $\left|m_{\beta \beta}\right|$ can be larger (smaller) than that predicted in the case of the exchange of light neutrinos with IH mass spectrum and $\left|m_{\beta \beta}\right|$ will exhibit a dependence on the atomic number $A$ of the decaying nucleus. It should be mentioned that, given the already high level of fine-tuning required 
for the cancellation between the tree-level and one-loop light neutrino contributions in $m_{\beta \beta}$, an additional cancellation between the light and heavy Majorana neutrino contributions would suggest further fine-tuning.

The main features of figure 3 also appear for larger splittings $\Delta M$. In particular, the necessity of fine-tuned cancellation between the tree-level and one-loop correction to the light neutrino mass matrix is present also in this case. The level of the fine-tuning required increases with $M_{1}$, as we will show in section 4 .

\subsection{Radiative corrections to the ESS and ISS scenarios}

In this section, we compute the one-loop contribution to the effective Majorana neutrino mass in the ESS and ISS limits of the seesaw Lagrangian (2.1) with two RH neutrinos. Accordingly, we apply the parametrization of the Dirac and Majorana mass matrices reported in eq. (2.33) to the general expression given in eq. (3.2). The exact result of the one-loop contribution in terms of the parameters introduced in (2.33) is reported in appendix A.

For the ESS scenario we have at leading order in $\Lambda / \mu^{\prime}$

$$
m_{\beta \beta}^{1-\text { loop }} \approx \frac{\mu^{\prime}}{2} \frac{y_{1 e}^{2}}{(4 \pi)^{2}}\left(\frac{3 \ln \left(\mu^{\prime 2} / M_{Z}^{2}\right)}{\mu^{\prime 2} / M_{Z}^{2}-1}+\frac{\ln \left(\mu^{\prime 2} / M_{H}^{2}\right)}{\mu^{\prime 2} / M_{H}^{2}-1}\right) .
$$

Notice that for $\mu^{\prime} \gg M_{H}, M_{Z}$, this expression reduces to

$$
m_{\beta \beta}^{1-\text { loop }} \approx \frac{y_{1 e}^{2}}{(4 \pi)^{2}}\left(\frac{3 M_{Z}^{2}}{2 \mu^{\prime}} \ln \left(\mu^{\prime 2} / M_{Z}^{2}\right)+\frac{M_{H}^{2}}{2 \mu^{\prime}} \ln \left(\mu^{\prime 2} / M_{H}^{2}\right)\right) .
$$

Therefore, when $\mu^{\prime} \gg M_{H}, M_{Z}$, since the lepton number violating scale $\mu^{\prime}$ is introduced at high energies, the one-loop contribution to the light neutrino masses appears to be suppressed as $1 / \mu^{\prime}$, as expected.

In the ISS realisation, i.e. for $\epsilon v, \mu, \mu^{\prime} \ll \Lambda$, we obtain

$$
\begin{aligned}
m_{\beta \beta}^{1-\text { loop }} \approx & \frac{1}{(4 \pi)^{2}}\left(\epsilon \Lambda y_{1 e} y_{2 e}-\frac{\mu}{2} y_{1 e}^{2}\right)\left(\frac{3 \ln \left(\Lambda^{2} / M_{Z}^{2}\right)}{\Lambda^{2} / M_{Z}^{2}-1}+\frac{\ln \left(\Lambda / M_{H}^{2}\right)}{\Lambda^{2} / M_{H}^{2}-1}\right) \\
& -\frac{\mu+\mu^{\prime}}{2} \frac{y_{1 e}^{2}}{(4 \pi)^{2}}\left(\frac{4 M_{H}^{2} M_{Z}^{2}-\Lambda^{2}\left(M_{H}^{2}+3 M_{Z}^{2}\right)}{\left(\Lambda^{2}-M_{Z}^{2}\right)\left(\Lambda^{2}-M_{H}^{2}\right)}+\frac{\ln \left(\Lambda^{2} / M_{H}^{2}\right)}{\left(\Lambda^{2} / M_{H}^{2}-1\right)^{2}}+\frac{3 \ln \left(\Lambda^{2} / M_{Z}^{2}\right)}{\left(\Lambda^{2} / M_{Z}^{2}-1\right)^{2}}\right) .
\end{aligned}
$$

It is remarkable that in the ESS limit with $\mu^{\prime} \lesssim M_{H}, M_{Z}$ and in the ISS limit the oneloop correction to the light neutrino masses has a contribution proportional to $\mu^{\prime}$. This dependence on $\mu^{\prime}$ is very relevant since at one-loop the light neutrino contribution to the $0 \nu \beta \beta$ decay amplitude does depend directly on $\mu^{\prime}$, as for the heavy contribution in (2.41) and (2.45). This makes much more difficult to obtain a dominant contribution from the $\mathrm{RH}$ neutrinos in this limit, unless a fine-tuning of the seesaw parameters is introduced to guarantee the smallness of the neutrino masses as it was indeed already shown in figure 3 .

\section{Large heavy neutrino contribution to $0 \nu \beta \beta$ decay}

In this section, we will address in more detail the question if the $\mathrm{RH}$ neutrinos can eventually give a sizable contribution to the $0 \nu \beta \beta$ decay rate. As we have already mentioned, 
cosmological constraints close the mass window of $M<100 \mathrm{MeV}[7,8]$ and thus only if the $\mathrm{RH}$ neutrino masses are larger than $100 \mathrm{MeV}$, a direct contribution to the process of interest can be expected.

Following the notation in ref. [5], the $0 \nu \beta \beta$ decay rate can be written as

$$
\frac{\Gamma_{0 \nu \beta \beta}}{\ln 2}=G_{01}\left|\sum_{j} U_{e j}^{2} \frac{m_{j}}{m_{e}} \mathcal{M}^{0 \nu \beta \beta}\left(m_{j}\right)\right|^{2},
$$

where $G_{01}$ is a well-known kinematic factor, $U$ is the unitary matrix given in eq. (2.4) which diagonalizes the complete neutrino mass matrix, $m_{j}$ are the corresponding eigenvalues, i.e., the neutrino masses (light and heavy), and $\mathcal{M}^{0 \nu \beta \beta}$ are the Nuclear Matrix Elements (NMEs) associated with the process. Notice that the NMEs depend on the mass of the neutrino mediating the process since the dependence on the neutrino propagator is already included in the NMEs computation. The sum should be made over all the neutrino masses, including the heavy ones. In the following we will use the NMEs data provided in [5]. In particular, we will consider the NMEs computed for the ${ }^{76} \mathrm{Ge}$. However, we have checked that the conclusions of our analysis do not significantly change considering a different nucleus.

We will use the modified Casas-Ibarra parametrization of the active-sterile neutrino mixing given in eq. (3.16), to compute the full effective Majorana neutrino mass $m_{\beta \beta}$, which is given by the sum of the contributions from the exchange of the light and heavy Majorana neutrinos. In this way, we include in the computation the effect of the oneloop correction to the light neutrino masses, reproducing at the same time the correct neutrino oscillation parameters. We will also take into account the relevant bounds on the active-sterile mixing which come from direct searches, charged lepton flavour violation and non-unitarity constraints [13-20]. Notice that the inclusion of such bounds guarantees the perturbativity of the neutrino Yukawa couplings for any value of $\mathrm{RH}$ neutrino masses considered in this paper.

In the top panels (down panels) of figures 4 and 5, the blue shaded area corresponds to the region of the parameter space in which $10^{-2} \mathrm{eV}<\left|m_{\beta \beta}^{\text {light }}+m_{\beta \beta}^{\text {heavy }}\right|<0.5 \mathrm{eV}$ $\left(10^{-2} \mathrm{eV}<\left|m_{\beta \beta}^{\text {heavy }}\right|<0.5 \mathrm{eV}\right)$, projected on the $\gamma-\Delta M$ plane for $\mathrm{NH}$ (IH) and several values of $M_{1}$. In these plots we have fixed the already measured PMNS parameters and neutrino oscillation mass differences to the best fit values given in [37]. The relevant Majorana and Dirac CP violation phases in the PMNS matrix have been set to zero, but we have checked that there is no significant impact on the results when other values are considered. The Casas-Ibarra parameter $\theta_{45}$ is also set to zero. It is irrelevant when the heavy Majorana neutrino exchange contribution is dominant (subdominant) in $m_{\beta \beta}$, but can play an important role in the interplay of the light and heavy Majorana neutrino exchange contributions when these two contributions are comparable in size [10]. The Higgs mass has been fixed to $M_{H}=125 \mathrm{GeV}$. The solid black line stands for different values, stated in the plots, of the $\alpha$ parameter defined as

$$
\alpha \equiv\left|m_{\beta \beta}^{1-\text { loop }}\right| /\left|m_{\beta \beta}^{\text {light }}\right|,
$$



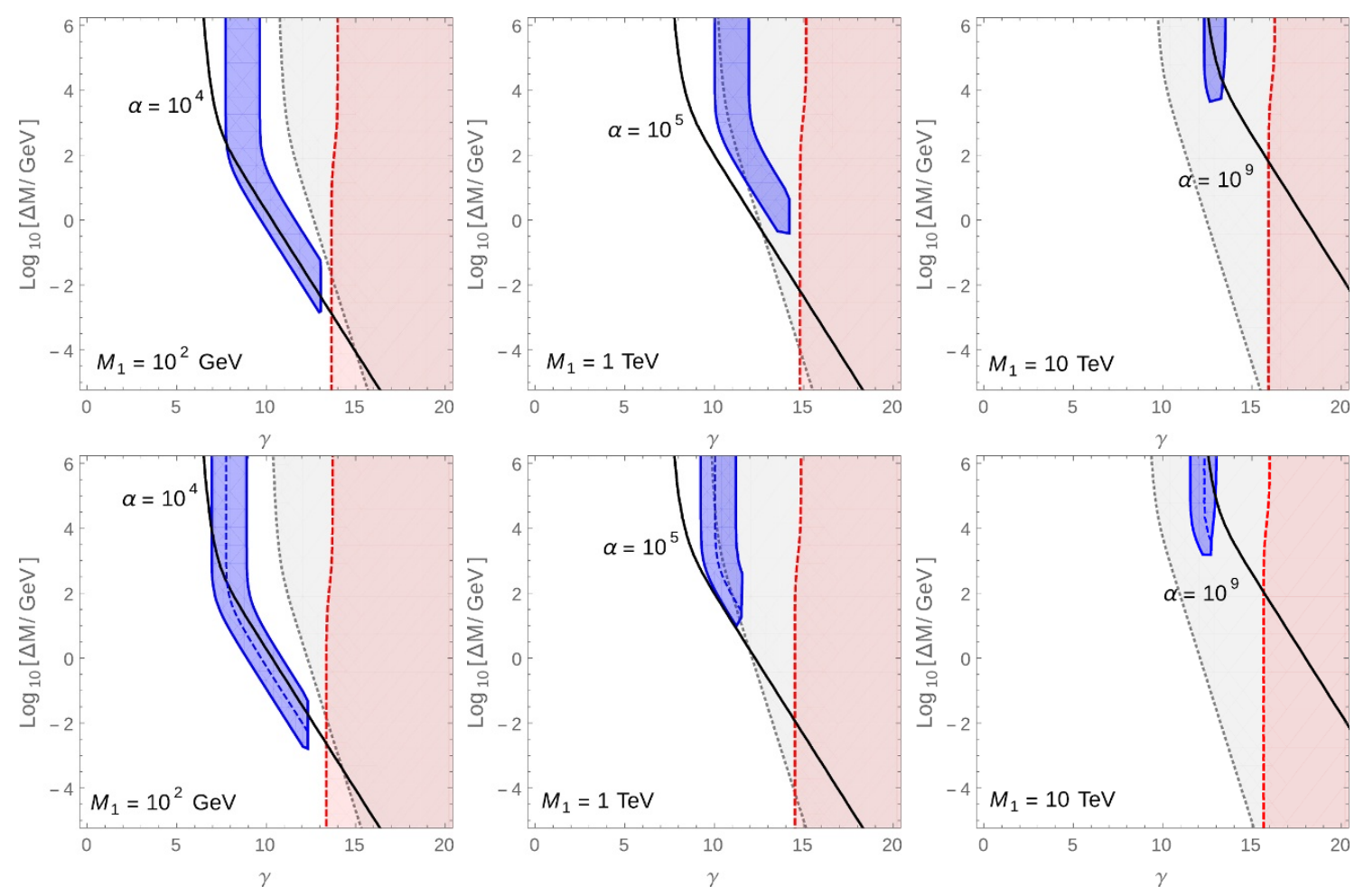

Figure 4. Neutrinoless double beta decay $\left(M_{1} \geq 100 \mathrm{GeV}\right)$. The blue shaded areas in the top panels (down panels) represent the region of the parameter space in which we have $10^{-2} \mathrm{eV}<$ $\left|m_{\beta \beta}^{\text {light }}+m_{\beta \beta}^{\text {heavy }}\right|<0.5 \mathrm{eV}\left(10^{-2} \mathrm{eV}<\left|m_{\beta \beta}^{\text {heavy }}\right|<0.5 \mathrm{eV}\right)$ in the case of $\mathrm{NH}$ (IH) neutrino mass spectrum with the active-sterile mixing (or couplings) $(\theta V)_{\ell k}$ satisfying the bounds form direct searches, charged lepton flavour violation and non-unitarity constraints. The black solid line stands for different values of the parameter $\alpha \equiv\left|m_{\beta \beta}^{1-\text { loop }}\right| /\left|m_{\beta \beta}^{\text {light }}\right|$, which quantifies the fine-tuning required in order to achieve the cancellation between the one-loop and tree-level contributions to the light neutrino masses. In the region to the right of the red dashed line the ratio between the leading order and the next to leading order contributions to the light neutrino masses in the seesaw expansion is smaller than 10. The gray region to the right of the dotted line corresponds to $y_{1 e}^{2} m_{\beta \beta}^{1-\text { loop }}>16 \pi^{2} m_{\beta \beta}^{\text {light }}$. The blue dashed line corresponds to $\left|m_{\beta \beta}^{\text {heavy }}\right|=0.05 \mathrm{eV}$. The measured neutrino oscillation parameters are fixed to the central values reported in [37].

where $m_{\beta \beta}^{\text {light }}=m_{\beta \beta}^{\text {tree }}+m_{\beta \beta}^{1-\text { loop }}$ is the full (tree-level plus one-loop) contribution to $m_{\beta \beta}$ given by the light neutrinos. Therefore, $\alpha$ quantifies the level of fine-tuning in the cancellation between $m_{\beta \beta}^{\text {tree }}$ and $m_{\beta \beta}^{1-\text { loop }}$ described in section 3.2 and required in order to keep the light neutrino masses and mixing to the observational values. Notice that the level of fine-tuning increases with $\alpha$. The region to the right of the black solid line corresponds to values of $\alpha$ larger than those stated in the plots.

In the red shaded area of figures 4 and 5, the ratio between the leading and next to leading order contributions to the light neutrino masses in the seesaw expansion is smaller than 10. The next to leading order contribution is given by [61]:

$$
\delta m_{\nu}=-\frac{1}{2}\left(m_{\nu}^{\text {tree }}+m_{\nu}^{\text {loop }}\right)(\theta V)(\theta V)^{\dagger}-\frac{1}{2}(\theta V)^{*}(\theta V)^{T}\left(m_{\nu}^{\text {tree }}+m_{\nu}^{\text {loop }}\right) .
$$


From this expression, one can conclude that a cancellation between the one-loop and treelevel contributions to the light neutrino masses remains at next to leading order in the seesaw expansion. This is in agreement with figures 4 and 5 , which show that the next to leading order contribution is always negligible in the range of parameters of interest.

Ignoring for the time being the impact of the two-loop corrections, which will be commented below, two main conclusions can be extracted from figures 4 and 5 . First, we have proved that a sizable and dominant heavy neutrino contribution to the $0 \nu \beta \beta$ decay is possible for $\mathrm{RH}$ neutrino masses as heavy as $10 \mathrm{TeV}$, satisfying at the same time the relevant constraints and keeping under control the light neutrino mass and mixing pattern. Second, and not less important, it is shown that this possibility can only take place if a highly fine-tuned cancellation between the tree-level and one-loop light neutrino masses is at work. The level of fine-tuning ranges from $\alpha=10^{4}$ to $10^{9}$, for heavy masses between $M_{1}=100 \mathrm{GeV}$ and $M_{1}=10 \mathrm{TeV}$. On the other hand, the level of fine-tuning is smaller for lighter masses, being in the case of $M_{1}=100 \mathrm{MeV}$ smaller than $\alpha=2$. In addition, we have checked that for $M_{1} \gtrsim 10 \mathrm{TeV}$ a heavy contribution to $m_{\beta \beta}$ in the range of sensitivity of the next-generation of experiments, $\left|m_{\beta \beta}\right| \gtrsim 0.01 \mathrm{eV}$, cannot be expected.

Figures 4 and 5 also show that in the limit $\Delta M \gg M_{1}$ the sizable heavy neutrino contribution corresponding to the blue region becomes independent of $\Delta M$, according with the ESS limit - see eq. (2.32). However, in the ISS limit $\Delta M \ll M_{1}$ this is not the case and, according to eq. (2.32), the smaller the heavy splitting $\Delta M$, the larger is the value of $\gamma$.

Notice that in the IH case we have plotted only $m_{\beta \beta}^{\text {heavy }}$ because $\left|m_{\beta \beta}^{\text {light }}\right|$ is already in the planned range of sensitivity of the next generation of $0 \nu \beta \beta$ decay experiments. In this case for $M_{1} \lesssim 10^{3} \mathrm{GeV}$ and $\Delta M \ll M_{1,2}$, there can be, in principle, a significant interplay between the light and heavy Majorana neutrino exchange contributions in the effective Majorana mass, as discussed at tree level in detail in [10] and summarised by us at the end of subsection II.A (see the paragraph before the last in subsection II.A). More specifically, due to this interplay of the light and heavy Majorana neutrino contributions, $\left|m_{\beta \beta}\right|$ can be larger (smaller) than that predicted in the case of the exchange of light neutrinos with IH mass spectrum and $\left|m_{\beta \beta}\right|$ will exhibit a dependence on the atomic number $A$ of the decaying nucleus. This can happen roughly in the region located to the left of the blue dashed line corresponding to $\left|m_{\beta \beta}^{\text {heavy }}\right|=0.05 \mathrm{eV}$ inside the blue areas in figures 4 and 5 .

In the $\mathrm{NH}$ case, the light neutrino contribution is smaller than $10^{-2} \mathrm{eV}$ and therefore any sizable effect to the process is due to the heavy neutrinos. This is why in the NH case we plot the total contribution $m_{\beta \beta}$, including light and heavy neutrinos.

It follows from figure 4 that for $M_{1} \geq 100 \mathrm{GeV}$ the regions of interest (the blue shaded areas) correspond to $\gamma \gtrsim 6$. For such values of $\gamma$, as it is not difficult to show, we have for the $\mathrm{NH}$ and $\mathrm{IH}$ neutrino mass spectra:

$$
\begin{aligned}
M_{1}\left|(\theta V)_{e 1}\right|^{2} & \approx M_{2}\left|(\theta V)_{e 2}\right|^{2} \\
& \approx \frac{e^{2 \gamma}}{4}\left|U_{e 2} \sqrt{m_{2}}-i U_{e 3} \sqrt{m_{3}}\right|^{2}, \quad \mathrm{NH} \\
& \approx \frac{e^{2 \gamma}}{4}\left|U_{e 1} \sqrt{m_{1}}-i U_{e 2} \sqrt{m_{2}}\right|^{2} . \mathrm{IH}
\end{aligned}
$$



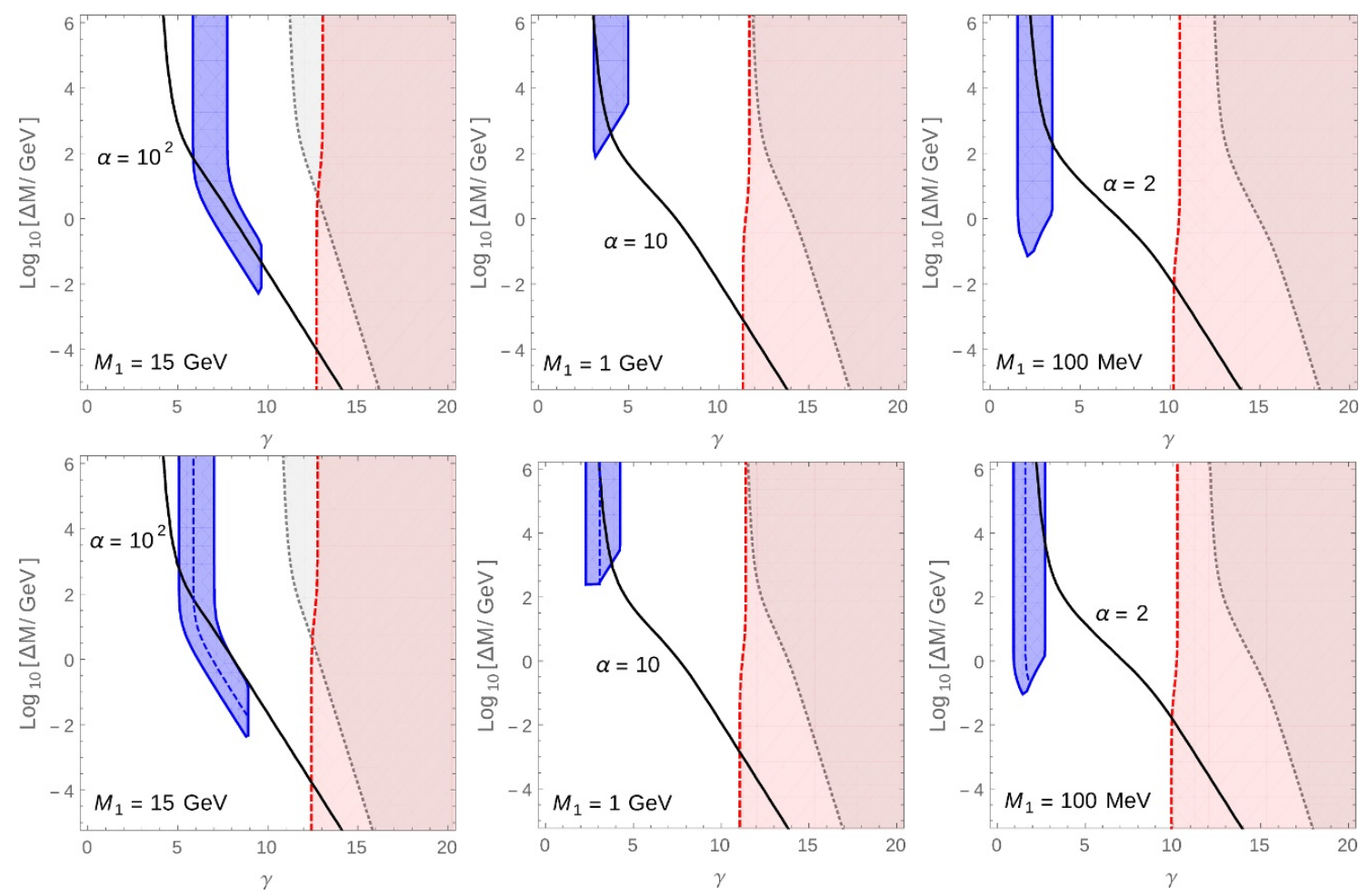

Figure 5. Neutrinoless double beta decay $\left(M_{1}<100 \mathrm{GeV}\right)$. The same conventions as in figure 4, but for different choices of $M_{1}$.

Taking into account that figure 4 is obtained by setting to zero the phase $\theta_{45}$ and the Dirac and Majorana phases in the PMNS matrix and by using the best fit values of the neutrino oscillation parameters, eqs. (4.4) and (4.5) imply the following relations between $\left|(\theta V)_{e 1(e 2)}\right|^{2}$ and the parameter $\gamma$ :

$$
M_{1}\left|(\theta V)_{e 1}\right|^{2} \approx M_{2}\left|(\theta V)_{e 2}\right|^{2} \approx e^{2 \gamma} 0.94(12.4) \times 10^{-3} \mathrm{eV}, \quad \mathrm{NH}(\mathrm{IH}) .
$$

In view of the high level of fine-tuning required in order to have a cancellation between the tree-level and one-loop light neutrino masses, the obvious question arising here is what is the role of the two-loop corrections. Can the two-loop corrections spoil this fine-tuned cancellation? In order to answer this question, we estimate the impact of the two-loop contributions. Since we are studying the case in which heavy neutrinos can give a sizable contribution to the $0 \nu \beta \beta$ decay, which means relatively large Yukawa couplings, we expect the diagram with two Higgs bosons in the loop to be the leading two-loop contribution to the light neutrino mass matrix. The contribution of this diagram can be roughly estimated as

$$
m_{\beta \beta}^{2-\text { loop }} \sim \frac{y_{1 e}^{2}}{(4 \pi)^{2}} m_{\beta \beta}^{1-\text { loop }}
$$

where $m_{\beta \beta}^{1-\text { loop }}$ is the one-loop contribution in $m_{\beta \beta}^{\text {light }}$. This estimate of the impact of the two loop corrections is also shown in figures 4 and 5 , where the gray area to the right of the dotted line corresponds to the region of the parameter space with $y_{1 e}^{2} m_{\beta \beta}^{1-\text { loop }}>16 \pi^{2} m_{\beta \beta}^{\text {light }}$. 
This region of the parameter space is excluded since the two-loop correction, which would dominate the light neutrino masses, would be larger than the value dictated by neutrino oscillation data. Notice that this would essentially exclude the possibility of having a large sterile neutrino contribution for $M_{1} \gtrsim 1 \mathrm{TeV}$, as can be seen in figures 4 and 5 . For $M_{1} \lesssim 100 \mathrm{GeV}$ the impact of the two-loop correction is basically negligible.

\section{Conclusions}

We have performed a systematic analysis of the radiative corrections to the light neutrino masses arising in low scale type I seesaw scenarios, where the $\mathrm{RH}$ (sterile) neutrino masses vary in the interval $100 \mathrm{MeV} \lesssim M \lesssim 10 \mathrm{TeV}$. Within this range of masses a significant enhancement of the neutrinoless double beta $(0 \nu \beta \beta)$ decay rate in several isotopes - at the level of sensitivity of the present and next generation experiments searching for this rare process - is possible, due to the new physics contribution in the decay amplitude given by the exchange of the virtual heavy sterile neutrinos. Notice that one of the most clear signatures of a significant heavy sterile Majorana neutrino contribution to the $0 \nu \beta \beta$ decay amplitude is the dependence of the $0 \nu \beta \beta$ decay effective Majorana mass, $\left|m_{\beta \beta}\right|$, on the atomic number $A$ of the decaying nucleus [12].

The requirement of a sizable contribution of heavy neutrinos with masses $\gtrsim 1 \mathrm{GeV}$ to the $0 \nu \beta \beta$ decay implies strong cancellations between the tree-level and one-loop expressions in the light neutrino mass matrix $m_{\nu}$ originated from the seesaw mechanism. We show that such a cancellation can always be achieved while being consistent with neutrino oscillation data and low energy constraints from direct searches, charged lepton flavour violation and non-unitarity by using a generalisation of the Casas-Ibarra parametrization of the neutrino Yukawa matrix, which can be derived from eqs. (3.15) and (3.16). We clarify the connection between this parametrization and the lepton number breaking terms in the seesaw Lagrangian, as usually defined in extended as well as inverse/direct seesaw UV completions of the Standard Model. Then, we numerically quantify the level of fine-tuning between the tree-level and one-loop parts of $m_{\nu}$ in the case the heavy neutrino contribution

$m_{\beta \beta}^{\text {heavy }}$ to the effective Majorana neutrino mass - which enters in the $0 \nu \beta \beta$ decay amplitude - is sizable, namely $\left|m_{\beta \beta}^{\text {heavy }}\right| \gtrsim 0.01 \mathrm{eV}$.

The main results of our analysis are summarised in figures 4 and 5, where we show that a fine-tuning of one part in $10^{4}\left(10^{5}\right)$ for RH neutrino masses $\sim 100(1000) \mathrm{GeV}$ is unavoidable in order to have an observable effect in $0 \nu \beta \beta$ decay experiments. Furthermore, we conclude that for seesaw scales $M$ larger than few $\mathrm{TeV}$, two-loop effects in the generation of the light neutrino masses cannot be neglected, thus excluding the possibility of having a large $\left|m_{\beta \beta}^{\text {heavy }}\right|$. Conversely, in the low mass regime, $M \lesssim 1 \mathrm{GeV}$, the level of fine-tuning in the seesaw parameter space is very mild and the sterile neutrino contribution can easily exceed the current limits on the effective Majorana neutrino mass.

Finally, we can conclude on the basis of the results obtained in the present analysis that $0 \nu \beta \beta$ sets the strongest constraints on lepton number violation in low scale type I seesaw extensions of the Standard Model. In particular, this implies a strong suppression 
of processes which involve the production at colliders (LHC included) of RH neutrinos and their decays with two like-sign charged leptons in the final state (see, e.g., [9, 62]).

\section{Acknowledgments}

The work of J.L.P. and S.T.P. was supported in part by the European Union FP7 ITN INVISIBLES (Marie Curie Actions, PITN-GA-2011-289442-INVISIBLES), by the INFN program on Theoretical Astroparticle Physics (TASP) and by the research grant 2012CPPYP7 ( Theoretical Astroparticle Physics) under the program PRIN 2012 funded by the Italian Ministry of Education, University and Research (MIUR). S.T.P. acknowledges partial support from the World Premier International Research Center Initiative (WPI Initiative), MEXT, Japan.

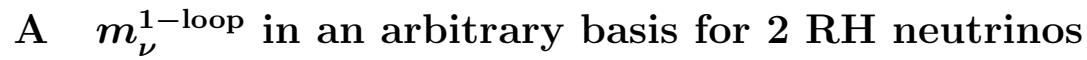

We report in this appendix the full computation of the one-loop correction (3.2) to the light neutrino mass matrix in terms of the seesaw parameters introduced in eq. (2.33), from which it is possible to derive the one-loop correction to effective Majorana neutrino mass in the extended and inverse seesaw limits, eqs. (3.17) and (3.19), respectively. In order to obtain an analytic expression for the one-loop neutrino mass matrix, we conveniently change the basis of the heavy RH neutrinos, i.e. $\nu_{a R}=\hat{V}_{a b} \nu_{b R}^{\prime}$, with the unitary transformation

$$
\hat{V}=\frac{1}{\sqrt{2}}\left(\begin{array}{cc}
i & 1 \\
-i & 1
\end{array}\right)
$$

In the new basis the $\mathrm{RH}$ neutrino Majorana mass matrix takes the form:

$$
M_{R}^{\prime} \equiv \hat{V}^{T} M_{R} \hat{V}=\frac{1}{2}\left(\begin{array}{cc}
2 \Lambda-\left(\mu+\mu^{\prime}\right) & -i\left(\mu-\mu^{\prime}\right) \\
-i\left(\mu-\mu^{\prime}\right) & 2 \Lambda+\left(\mu+\mu^{\prime}\right)
\end{array}\right)
$$

Then, the resulting one-loop Majorana mass term for active neutrinos is

$$
m_{\nu}^{1-\text { loop }}=\frac{1}{(4 \pi v)^{2}} m_{D} \hat{V}\left(M_{R}^{\prime-1} F\left(M_{R}^{\prime} M_{R}^{\prime}{ }^{\dagger}\right)+F\left(M_{R}^{\prime \dagger} M_{R}^{\prime}\right) M_{R}^{\prime-1}\right) \hat{V}^{T} m_{D}^{T},
$$

where the loop function $F(x)$ is defined in eq. (3.3) and the Dirac mass matrix $m_{D}$ is parametrized as in (2.33). In this case we have: ${ }^{9}$

$$
\begin{aligned}
& M_{R}^{\prime} M_{R}^{\dagger}=\left(\Lambda^{2}+\frac{1}{2}\left(|\mu|^{2}+\left|\mu^{\prime}\right|^{2}\right)\right)\left(\mathbf{1}_{\mathbf{2} \times \mathbf{2}}-A(a, b, c)\right), \\
& M_{R}^{\prime}{ }^{\dagger} M_{R}^{\prime}=\left(\Lambda^{2}+\frac{1}{2}\left(|\mu|^{2}+\left|\mu^{\prime}\right|^{2}\right)\right)\left(\mathbf{1}_{\mathbf{2} \times \mathbf{2}}-A(a,-b, c)\right),
\end{aligned}
$$

where

$$
A(a, b, c) \equiv\left(a \sigma_{\mathbf{3}}+b \sigma_{\mathbf{2}}+c \sigma_{\mathbf{1}}\right),
$$

\footnotetext{
${ }^{9}$ We assume without loss of generality that the parameter $\Lambda$ in (2.33) is real.
} 
$\sigma_{i}(i=1,2,3)$ denoting the $2 \times 2$ Pauli matrices. The real parameters $a, b$ and $c$ are defined as

$$
\begin{aligned}
a & =\frac{2 \Lambda \operatorname{Re}\left(\mu+\mu^{\prime}\right)}{2 \Lambda^{2}+|\mu|^{2}+\left|\mu^{\prime}\right|^{2}}, \\
b & =\frac{\left|\mu^{\prime}\right|^{2}-|\mu|^{2}}{2 \Lambda^{2}+|\mu|^{2}+\left|\mu^{\prime}\right|^{2}}, \\
c & =\frac{2 \Lambda \operatorname{Im}\left(\mu^{\prime}-\mu\right)}{2 \Lambda^{2}+|\mu|^{2}+\left|\mu^{\prime}\right|^{2}} .
\end{aligned}
$$

In this way, one can obtain a closed form for the logarithms which enter in eq. (A.3) through the loop function. Indeed, we have

$$
\log \left[\mathbf{1}_{\mathbf{2} \times \mathbf{2}}-A(a, b, c)\right]=-\sum_{n=1}^{\infty} \frac{1}{n} A(a, b, c)^{n},
$$

with

$$
\begin{aligned}
& {\left[A(a, b, c)^{n}\right]_{11}=\frac{1}{2}\left(a^{2}+b^{2}+c^{2}\right)^{\frac{1}{2}(-1+n)}\left(a\left(1-(-1)^{n}\right)+\sqrt{a^{2}+b^{2}+c^{2}}\left(1+(-1)^{n}\right)\right)} \\
& {\left[A(a, b, c)^{n}\right]_{22}=-\frac{1}{2}\left(a^{2}+b^{2}+c^{2}\right)^{\frac{1}{2}(-1+n)}\left(a\left(1-(-1)^{n}\right)-\sqrt{a^{2}+b^{2}+c^{2}}\left(1+(-1)^{n}\right)\right)} \\
& {\left[A(a, b, c)^{n}\right]_{12}=\left[A(a, b, c)^{n}\right]_{21}^{*}=\frac{i}{2}(b+i c)\left(a^{2}+b^{2}+c^{2}\right)^{\frac{1}{2}(-1+n)}\left(-1+(-1)^{n}\right) . \quad \text { A. } 11}
\end{aligned}
$$

Then, one can show that the infinite series in (A.10) gives the exact results

$$
\begin{aligned}
& {\left[\log \left[\mathbf{1}_{\mathbf{2} \times \mathbf{2}}-A(a, b, c)\right]\right]_{11}=\frac{2 a \tanh ^{-1}\left(\sqrt{a^{2}+b^{2}+c^{2}}\right)}{\sqrt{a^{2}+b^{2}+c^{2}}}-\log \left(1-a^{2}-b^{2}-c^{2}\right),} \\
& {\left[\log \left[\mathbf{1}_{\mathbf{2} \times \mathbf{2}}-A(a, b, c)\right]\right]_{12}=\left[\log \left(\mathbf{1}_{\mathbf{2} \times \mathbf{2}}-A(a, b, c)\right)\right]_{21}^{*}=\frac{i(b+i c) \log \left(\frac{1-\sqrt{a^{2}+b^{2}+c^{2}}}{1+\sqrt{a^{2}+b^{2}+c^{2}}}\right)}{\sqrt{a^{2}+b^{2}+c^{2}}},} \\
& {\left[\log \left[\mathbf{1}_{\mathbf{2} \times \mathbf{2}}-A(a, b, c)\right]\right]_{22}=-\frac{2 a \tanh ^{-1}\left(\sqrt{a^{2}+b^{2}+c^{2}}\right)}{\sqrt{a^{2}+b^{2}+c^{2}}}-\log \left(1-a^{2}-b^{2}-c^{2}\right)(\mathrm{A} .}
\end{aligned}
$$

Therefore, by replacing eqs. (A.12) in (A.3), we obtain an analytic expression for the one-loop contribution to the light Majorana neutrino mass matrix as a function of the parameters given in (2.33).

Open Access. This article is distributed under the terms of the Creative Commons Attribution License (CC-BY 4.0), which permits any use, distribution and reproduction in any medium, provided the original author(s) and source are credited.

\section{References}

[1] P. Minkowski, $\mu \rightarrow$ er at a Rate of One Out of $10^{9}$ Muon Decays?, Phys. Lett. B 67 (1977) 421 [INSPIRE]. 
[2] T. Yanagida, Proceedinds of the Workshop on Unified Theories and Baryon Number in the Universe, eds. A. Sawada and A. Sugamoto, Tsukuba, Japan (1979).

[3] M. Gell-Mann, P. Ramond and R. Slansky, Proceedings of the Supergravity Stony Brook Workshop, eds. P. Van Nieuwenhuizen and D. Freedman, New York (1979).

[4] R.N. Mohapatra and G. Senjanović, Neutrino Mass and Spontaneous Parity Violation, Phys. Rev. Lett. 44 (1980) 912 [INSPIRE].

[5] M. Blennow, E. Fernandez-Martinez, J. Lopez-Pavon and J. Menendez, Neutrinoless double beta decay in seesaw models, JHEP 07 (2010) 096 [arXiv:1005.3240] [INSPIRE].

[6] J. Lopez-Pavon, S. Pascoli and C.-f. Wong, Can heavy neutrinos dominate neutrinoless double beta decay?, Phys. Rev. D 87 (2013) 093007 [arXiv:1209.5342] [INSPIRE].

[7] P. Hernández, M. Kekic and J. Lopez-Pavon, Low-scale seesaw models versus $N_{\mathrm{eff}}$, Phys. Rev. D 89 (2014) 073009 [arXiv: 1311.2614] [INSPIRE].

[8] P. Hernández, M. Kekic and J. Lopez-Pavon, $N_{\text {eff }}$ in low-scale seesaw models versus the lightest neutrino mass, Phys. Rev. D 90 (2014) 065033 [arXiv:1406.2961] [InSPIRE].

[9] A. Ibarra, E. Molinaro and S.T. Petcov, TeV Scale See-Saw Mechanisms of Neutrino Mass Generation, the Majorana Nature of the Heavy Singlet Neutrinos and $(\beta \beta)_{0 \nu}$-Decay, JHEP 09 (2010) 108 [arXiv: 1007.2378] [inSPIRE].

[10] A. Ibarra, E. Molinaro and S.T. Petcov, Low Energy Signatures of the TeV Scale See-Saw Mechanism, Phys. Rev. D 84 (2011) 013005 [arXiv:1103.6217] [INSPIRE].

[11] M. Mitra, G. Senjanović and F. Vissani, Neutrinoless Double Beta Decay and Heavy Sterile Neutrinos, Nucl. Phys. B 856 (2012) 26 [arXiv:1108.0004] [InSPIRE].

[12] A. Halprin, S.T. Petcov and S.P. Rosen, Effects of Light and Heavy Majorana Neutrinos in Neutrinoless Double Beta Decay, Phys. Lett. B 125 (1983) 335 [INSPIRE].

[13] S. Antusch, C. Biggio, E. Fernandez-Martinez, M.B. Gavela and J. Lopez-Pavon, Unitarity of the Leptonic Mixing Matrix, JHEP 10 (2006) 084 [hep-ph/0607020] [INSPIRE].

[14] S. Antusch, J.P. Baumann and E. Fernandez-Martinez, Non-Standard Neutrino Interactions with Matter from Physics Beyond the Standard Model, Nucl. Phys. B 810 (2009) 369 [arXiv: 0807.1003] [INSPIRE].

[15] A. Atre, T. Han, S. Pascoli and B. Zhang, The Search for Heavy Majorana Neutrinos, JHEP 05 (2009) 030 [arXiv:0901.3589] [INSPIRE].

[16] R. Alonso, M. Dhen, M.B. Gavela and T. Hambye, Muon conversion to electron in nuclei in type-I seesaw models, JHEP 01 (2013) 118 [arXiv:1209.2679] [INSPIRE].

[17] S. Antusch and O. Fischer, Non-unitarity of the leptonic mixing matrix: Present bounds and future sensitivities, JHEP 10 (2014) 94 [arXiv:1407.6607] [INSPIRE].

[18] M. Drewes and B. Garbrecht, Experimental and cosmological constraints on heavy neutrinos, arXiv: 1502.00477 [INSPIRE].

[19] D.N. Dinh, A. Ibarra, E. Molinaro and S.T. Petcov, The $\mu-e$ Conversion in Nuclei, $\mu \rightarrow e \gamma, \mu \rightarrow 3 e$ Decays and TeV Scale See-Saw Scenarios of Neutrino Mass Generation, JHEP 08 (2012) 125 [Erratum ibid. 1309 (2013) 023] [arXiv:1205.4671] [INSPIRE].

[20] C.G. Cely, A. Ibarra, E. Molinaro and S.T. Petcov, Higgs Decays in the Low Scale Type I See-Saw Model, Phys. Lett. B 718 (2013) 957 [arXiv:1208.3654] [InSPIRE]. 
[21] J.A. Casas and A. Ibarra, Oscillating neutrinos and $\mu \rightarrow e \gamma$, Nucl. Phys. B 618 (2001) 171 [hep-ph/0103065] [INSPIRE].

[22] B. Pontecorvo, Mesonium and anti-mesonium, Sov. Phys. JETP 6 (1957) 429 [Zh. Eksp. Teor. Fiz. 33 (1957) 549].

[23] Z. Maki, M. Nakagawa and S. Sakata, Remarks on the unified model of elementary particles, Prog. Theor. Phys. 28 (1962) 870 [InSPIRE].

[24] A. Donini, P. Hernández, J. Lopez-Pavon, M. Maltoni and T. Schwetz, The minimal 3+2 neutrino model versus oscillation anomalies, JHEP 07 (2012) 161 [arXiv:1205.5230] [INSPIRE].

[25] M. Blennow and E. Fernandez-Martinez, Parametrization of Seesaw Models and Light Sterile Neutrinos, Phys. Lett. B 704 (2014) 223 [arXiv:1107.3992] [INSPIRE].

[26] R.N. Mohapatra, Mechanism for Understanding Small Neutrino Mass in Superstring Theories, Phys. Rev. Lett. 56 (1986) 561 [InSPIRE].

[27] R.N. Mohapatra and J.W.F. Valle, Neutrino Mass and Baryon Number Nonconservation in Superstring Models, Phys. Rev. D 34 (1986) 1642 [InSPIRE].

[28] G.C. Branco, W. Grimus and L. Lavoura, The Seesaw Mechanism in the Presence of a Conserved Lepton Number, Nucl. Phys. B 312 (1989) 492 [INSPIRE].

[29] A. Kleppe, Extending The Standard Model With Two Right-Handed Neutrinos, in Lohusalu, Neutrino physics (1995) 118-125.

[30] E. Ma, D.P. Roy and U. Sarkar, A Seesaw model for atmospheric and solar neutrino oscillations, Phys. Lett. B 444 (1998) 391 [hep-ph/9810309] [INSPIRE].

[31] P.H. Frampton, S.L. Glashow and T. Yanagida, Cosmological sign of neutrino CP-violation, Phys. Lett. B 548 (2002) 119 [hep-ph/0208157] [INSPIRE].

[32] M. Raidal and A. Strumia, Predictions of the most minimal seesaw model, Phys. Lett. B 553 (2003) 72 [hep-ph/0210021] [INSPIRE].

[33] V. Barger, D.A. Dicus, H.-J. He and T.-j. Li, Structure of cosmological CP-violation via neutrino seesaw, Phys. Lett. B 583 (2004) 173 [hep-ph/0310278] [INSPIRE].

[34] T. Endoh, S. Kaneko, S.K. Kang, T. Morozumi and M. Tanimoto, CP violation in neutrino oscillation and leptogenesis, Phys. Rev. Lett. 89 (2002) 231601 [hep-ph/0209020] [INSPIRE].

[35] A. Ibarra and G.G. Ross, Neutrino phenomenology: The Case of two right-handed neutrinos, Phys. Lett. B 591 (2004) 285 [hep-ph/0312138] [INSPIRE].

[36] A. Ibarra and G.G. Ross, Neutrino properties from Yukawa structure, Phys. Lett. B 575 (2003) 279 [hep-ph/0307051] [INSPIRE].

[37] M.C. Gonzalez-Garcia, M. Maltoni and T. Schwetz, Updated fit to three neutrino mixing: status of leptonic CP-violation, JHEP 11 (2014) 052 [arXiv:1409.5439] [INSPIRE].

[38] F. del Aguila, J.A. Aguilar-Saavedra and R. Pittau, Neutrino physics at large colliders, J. Phys. Conf. Ser. 53 (2006) 506 [hep-ph/0606198] [INSPIRE].

[39] M.B. Gavela, T. Hambye, D. Hernandez and P. Hernández, Minimal Flavour Seesaw Models, JHEP 09 (2009) 038 [arXiv:0906.1461] [INSPIRE].

[40] GERDA collaboration, M. Agostini et al., Results on Neutrinoless Double- $\beta$ Decay of ${ }^{76} \mathrm{Ge}$ 
from Phase I of the GERDA Experiment, Phys. Rev. Lett. 111 (2013) 122503 [arXiv: 1307.4720] [INSPIRE].

[41] EXO-200 collaboration, J.B. Albert et al., Search for Majorana neutrinos with the first two years of EXO-200 data, Nature $\mathbf{5 1 0}$ (2014) 229 [arXiv:1402.6956] [INSPIRE].

[42] KamLAND-Zen collaboration, A. Gando et al., Limit on Neutrinoless $\beta \beta$ Decay of ${ }^{136} X e$ from the First Phase of KamLAND-Zen and Comparison with the Positive Claim in ${ }^{76} G e$, Phys. Rev. Lett. 110 (2013) 062502 [arXiv:1211.3863] [INSPIRE].

[43] CUORE collaboration, K. Alfonso et al., Search for Neutrinoless Double-Beta Decay of ${ }^{130}$ Te with CUORE-0, Phys. Rev. Lett. 115 (2015) 102502 [arXiv: 1504.02454] [INSPIRE].

[44] Majorana collaboration, N. Abgrall et al., The Majorana Demonstrator Neutrinoless Double-Beta Decay Experiment, Adv. High Energy Phys. 2014 (2014) 365432 [arXiv: 1308.1633] [INSPIRE].

[45] SNO+ collaboration, J. Hartnell, Neutrinoless Double Beta Decay with SNO+, J. Phys. Conf. Ser. 375 (2012) 042015 [arXiv: 1201.6169] [INSPIRE].

[46] H. Bhang et al., AMoRE experiment: a search for neutrinoless double beta decay of Mo-100 isotope with Ca-40 MoO-100(4) cryogenic scintillation detector, J. Phys. Conf. Ser. $\mathbf{3 7 5}$ (2012) 042023 [INSPIRE].

[47] Particle Data Group collaboration, K.A. Olive et al., Review of Particle Physics, Chin. Phys. C 38 (2014) 090001 [INSPIRE].

[48] S. Pascoli and S.T. Petcov, Majorana Neutrinos, Neutrino Mass Spectrum and the $|<m>| \sim 10^{-3}$ eV Frontier in Neutrinoless Double Beta Decay, Phys. Rev. D 77 (2008) 113003 [arXiv:0711.4993] [INSPIRE].

[49] S. Pascoli, M. Mitra and S. Wong, Effect of cancellation in neutrinoless double beta decay, Phys. Rev. D 90 (2014) 093005 [arXiv: 1310.6218] [InSPIRE].

[50] S. Pascoli and S.T. Petcov, The SNO solar neutrino data, neutrinoless double beta decay and neutrino mass spectrum: Addendum, Phys. Lett. B 580 (2004) 280 [hep-ph/0310003] [INSPIRE].

[51] S. Pascoli and S.T. Petcov, The SNO solar neutrino data, neutrinoless double beta decay and neutrino mass spectrum, Phys. Lett. B 544 (2002) 239 [hep-ph/0205022] [INSPIRE].

[52] S.K. Kang and C.S. Kim, Extended double seesaw model for neutrino mass spectrum and low scale leptogenesis, Phys. Lett. B 646 (2007) 248 [hep-ph/0607072] [INSPIRE].

[53] C.N. Leung and S.T. Petcov, A Comment on the Coexistence of Dirac and Majorana Massive Neutrinos, Phys. Lett. B 125 (1983) 461 [INSPIRE].

[54] S.M. Bilenky and S.T. Petcov, Massive Neutrinos and Neutrino Oscillations, Rev. Mod. Phys. 59 (1987) 671 [Erratum ibid. 61 (1989) 169] [INSPIRE].

[55] L. Wolfenstein, Different Varieties of Massive Dirac Neutrinos, Nucl. Phys. B 186 (1981) 147 [INSPIRE].

[56] S.T. Petcov, On PseudoDirac Neutrinos, Neutrino Oscillations and Neutrinoless Double beta Decay, Phys. Lett. B 110 (1982) 245 [INSPIRE].

[57] A. Pilaftsis, Radiatively induced neutrino masses and large Higgs neutrino couplings in the standard model with Majorana fields, Z. Phys. C 55 (1992) 275 [hep-ph/9901206] [INSPIRE]. 
[58] W. Grimus and L. Lavoura, One-loop corrections to the seesaw mechanism in the multi-Higgs-doublet standard model, Phys. Lett. B 546 (2002) 86 [hep-ph/0207229] [INSPIRE].

[59] D. Aristizabal Sierra and C.E. Yaguna, On the importance of the 1-loop finite corrections to seesaw neutrino masses, JHEP 08 (2011) 013 [arXiv:1106.3587] [INSPIRE].

[60] P.S.B. Dev and A. Pilaftsis, Minimal Radiative Neutrino Mass Mechanism for Inverse Seesaw Models, Phys. Rev. D 86 (2012) 113001 [arXiv:1209.4051] [INSPIRE].

[61] W. Grimus and L. Lavoura, The Seesaw mechanism at arbitrary order: Disentangling the small scale from the large scale, JHEP 11 (2000) 042 [hep-ph/0008179] [INSPIRE].

[62] F. del Aguila and J.A. Aguilar-Saavedra, Distinguishing seesaw models at LHC with multi-lepton signals, Nucl. Phys. B $\mathbf{8 1 3}$ (2009) 22 [arXiv:0808.2468] [INSPIRE]. 